\title{
Transposable element insertions shape gene regulation and melanin production in a fungal pathogen of wheat
}

\author{
Parvathy Krishnan ${ }^{1}$, Lukas Meile ${ }^{1}$, Clémence Plissonneau ${ }^{1,2}$, Xin Ma ${ }^{1}$, Fanny E. Hartmann ${ }^{1,3}$, Daniel Croll ${ }^{1,4}$,
} Bruce A. McDonald ${ }^{1}$ and Andrea Sánchez-Vallet ${ }^{*}$ (D)

\begin{abstract}
Background: Fungal plant pathogens pose major threats to crop yield and sustainable food production if they are highly adapted to their host and the local environment. Variation in gene expression contributes to phenotypic diversity within fungal species and affects adaptation. However, very few cases of adaptive regulatory changes have been reported in fungi and the underlying mechanisms remain largely unexplored. Fungal pathogen genomes are highly plastic and harbor numerous insertions of transposable elements, which can potentially contribute to gene expression regulation. In this work, we elucidated how transposable elements contribute to variation in melanin accumulation, a quantitative trait in fungi that affects survival under stressful conditions.

Results: We demonstrated that differential transcriptional regulation of the gene encoding the transcription factor Zmr1, which controls expression of the genes in the melanin biosynthetic gene cluster, is responsible for variation in melanin accumulation in the fungal plant pathogen Zymoseptoria tritici. We show that differences in melanin levels between two strains of $Z$. tritici are due to two levels of transcriptional regulation: (1) variation in the promoter sequence of $Z m r 1$ and (2) an insertion of transposable elements upstream of the Zmr1 promoter. Remarkably, independent insertions of transposable elements upstream of Zmr1 occurred in 9\% of $Z$. tritici strains from around the world and negatively regulated Zmrl expression, contributing to variation in melanin accumulation.
\end{abstract}

Conclusions: Our studies identified two levels of transcriptional control that regulate the synthesis of melanin. We propose that these regulatory mechanisms evolved to balance the fitness costs associated with melanin production against its positive contribution to survival in stressful environments.

Keywords: Epiallele, Gene expression variation, Quantitative trait, Melanization, Fungal plant pathogen,

Transposable element, Wheat

\section{Background}

Understanding the genetic basis of adaptive traits is an important goal in ecology and evolutionary biology. Variation in gene expression is believed to underlie much of the phenotypic diversity within a species [1-3]. However, most of the adaptive mutations identified until now are in coding sequences [4-6]. The reason for this is that protein variants are easier to identify because the

\footnotetext{
*Correspondence: andrea.sanchez@usys.ethz.ch

'Plant Pathology, Institute of Integrative Biology, ETH Zurich, Zurich, Switzerland

Full list of author information is available at the end of the article
}

genetic code enables in silico prediction of causative mutations. The contribution of changes in gene regulation to phenotypic diversity and the mechanisms underlying these changes merit further investigation.

Mutations outside of coding regions can lead to alterations in transcription, splicing, transcript stability, or chromatin remodeling and consequently can affect the regulation of gene expression [6-8]. Modifications in cis-regulatory sequences, such as single nucleotide polymorphisms (SNPs) and indels, can affect their expression and are thought to be targets of evolution [9-14]. Additionally, insertions of transposable elements inside or

C) Sánchez-Vallet et al. 2018 Open Access This article is distributed under the terms of the Creative Commons Attribution 4.0 International License (http://creativecommons.org/licenses/by/4.0/), which permits unrestricted use, distribution, and reproduction in any medium, provided you give appropriate credit to the original author(s) and the source, provide a link to the Creative Commons license, and indicate if changes were made. The Creative Commons Public Domain Dedication waiver (http://creativecommons.org/publicdomain/zero/1.0/) applies to the data made available in this article, unless otherwise stated. 
outside the promoters may introduce elements that enhance or repress transcription and induce changes in the chromatin state of adjacent regions, thus altering the expression of nearby genes [15-21]. Thus, transposable element insertions have the potential to contribute to phenotypic diversity through chromatin remodeling and regulation of gene expression.

In fungi, rapidly evolving regions, which frequently contain genes involved in virulence and stress tolerance, are often associated with transposable elements [22]. The contributions of transposable elements to evolution of adjacent regions and their effects on fungal diversity are frequently postulated [22-24] but have rarely been demonstrated. Many fungal plant pathogens are broadly distributed across the globe and are exposed to constantly fluctuating climatic conditions, a wide range of fungicides, and host immune defenses which can vary according to the host plant genotype [25, 26]. Adaptation to changing environments typically favors the capacity to respond rapidly to stress. Additionally, populations that maintain a high standing genetic variation for adaptive traits are typically more successful in surviving in changing environments [27]. One such adaptive trait is melanization. Melanin is a broadly distributed secondary metabolite required by many fungi for host colonization and survival under stress conditions [28-31]. Two major types of melanin have been extensively described in fungi, namely dihydroxynaphthalene (DHN) and dihydroxyphenylalanine melanin [32-34]. A high diversity in melanin accumulation among individuals within a species provides a mechanism for differential tolerance to rapidly changing and locally hazardous conditions [31].

Zymoseptoria tritici is a major wheat pathogen that has been extensively investigated for its potential to colonize different wheat cultivars and survive under stressful conditions, including exposure to high temperatures and fungicides [33, 35-37]. Z. tritici is known to have a plastic genome that includes numerous transposable element insertions ( $17 \%$ of the genome) and in which chromosomal rearrangements frequently occur $[36,38,39]$. It is thought that this genome plasticity can make important contributions to phenotypic variability, but the precise mechanisms underlying this phenomenon are not fully understood [36, 37, 40].

In four Swiss strains of $Z$. tritici, variable levels of melanin accumulation were observed and were postulated to contribute to differences in tolerance against abiotic stress, including fungicide resistance [33, 41]. We aimed to further explore the genetic basis of differences in melanin accumulation by using a previously performed genetic mapping approach [33]. A single quantitative trait locus (QTL) was identified that contained part of the polyketide synthase 1 (Pks1) gene cluster, which is involved in biosynthesis of DHN melanin in other fungal species [32, 34, 42, 43]. In this work, we remapped the QTL to the genome of one of the parental strains and we obtained a narrower and shifted QTL confidence interval, which allowed us to determine the genetic basis of the differences in melanin accumulation. We show that variation in gene expression, instead of variation in the coding sequence, underlies the observed differences in melanin accumulation. Variation in expression of a single gene, encoding the homolog of the transcription factor Cmr1 (Colletotrichum melanin regulation 1), which we named Zmr1 (for Zymoseptoria melanin regulation 1), explained the variation in melanization. We discovered two independent causes of variation in gene expression, namely SNPs in the promoter of $Z m r 1$ and an insertion of transposable elements upstream of the Zmrl promoter. We then showed that diversity in melanin accumulation at the species level is determined in part by independent insertions of transposable elements, which regulate $Z m r 1$ expression. We showed that melanin has a deleterious effect when the fungus is grown under optimal conditions but is beneficial in the presence of fungicides. We hypothesize that the complex regulation of $Z m r 1$ facilitates the emergence and maintenance of diversity in melanization to optimize a trade-off between the deleterious effect of melanin on the growth rate and its favorable effects on survival in stressful environments.

\section{Results}

\section{Differences in melanin accumulation are determined by the Pks1 cluster}

Melanin accumulation in the Swiss Z. tritici strain 3D1 was lower than that in the strain 3D7 at early time points (10 days post inoculation, dpi). The differences in melanization were temporal, as the lighter strain 3D1 accumulated similar amounts of melanin as 3D7 at later developmental stages (11-12 dpi; Fig. 1a, Additional file 1). We explored the genetic basis of these differences in melanin accumulation by using the previously obtained QTL for these two strains [33]. To narrow down the confidence interval, a new genetic map was obtained by using the completely assembled genome of the parental strain 3D7 [39] instead of the genome of the reference strain IPO323. This strategy provided us with approximately 10 times more SNP markers and enabled us to identify additional crossover events. The newly mapped 95\% confidence interval of the melanization QTL was narrowed from 43429 to $18135 \mathrm{bp}$ and contained six genes instead of 12 . The new QTL position shifted with respect to the earlier position, with an overlapping region of only $9299 \mathrm{bp}$. The region shared between the two QTLs contained the promoter of a gene encoding the homolog of the transcription factor Cmr1 (Colletotrichum melanin regulation 1), 


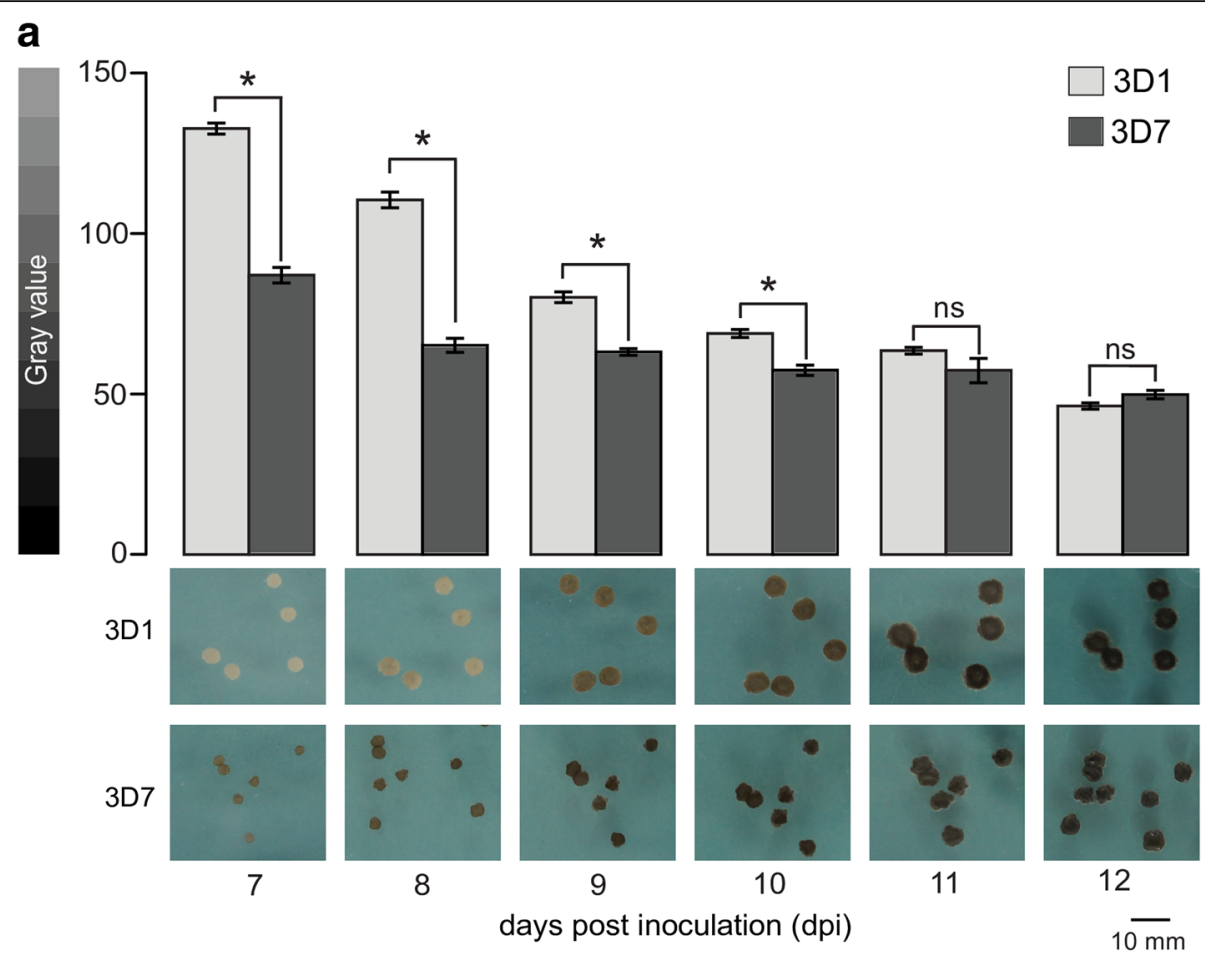

b

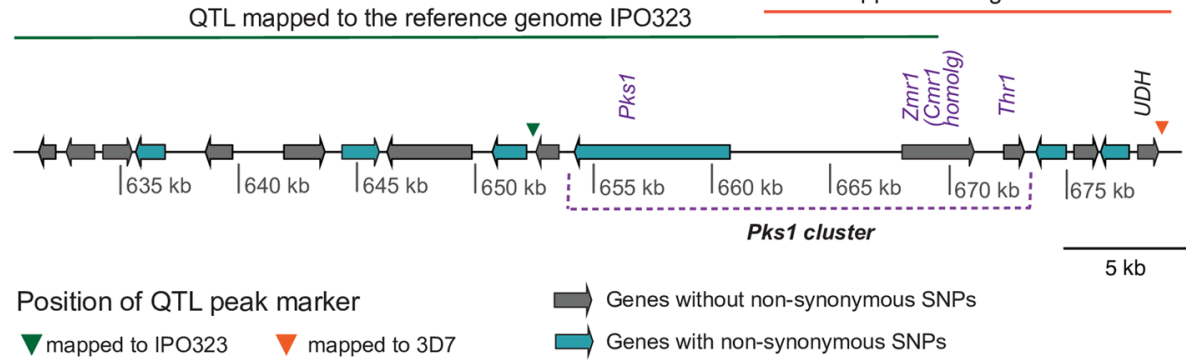

Fig. 1 Variability in melanization levels between 3D1 and 3D7 maps to the Pks 1 cluster. a The Swiss strain 3D1 is less melanized than 3D7. Melanization levels of 3D1 and 3D7 at 7 to 12 days. Bars represent standard errors of the mean gray value based on at least 60 colonies. Asterisks indicate significant differences according to Kruskal-Wallis test ( $p$ value $\leq 0.05$; ns non-significant). Representative pictures of both strains are shown below the bar plot for all the time points. The experiment was performed three times with similar results. Gray value scale ( $0=$ black, $255=$ white) is shown on the left. b Genes in the 95\% confidence interval of the QTL mapped to the genomes of the reference strain IPO323 and of the parental Swiss strain 3D7. The shift in the position of the QTL, genes with and without non-synonymous mutations, and the positions of the QTL peaking markers are indicated

which we named Zmr1 (for Zymoseptoria melanin regulation 1). Two of the genes within the new confidence interval belonged to the $P k s 1$ cluster, namely $Z m r 1$ and 1,3,8-trihydroxynaphthalene reductase (Thr1, Fig. 1b, Additional files 2 and 3 ).

\section{A transposable element insertion in the Pks1 gene cluster occurs only in the less melanized strain}

The most obvious candidate genes to explain differential melanin accumulation in the two parental strains were Zmr1 and Thr1. Both encoded proteins were identical between the parental strains (Additional file 3) and no mutations were detected in the promoter (1000 bp upstream of the start codon) of Thr1. However, 12 SNPs were identified in the promoter of $\mathrm{Zmrl}$ (Fig. 2a) and we hypothesized that these SNPs could explain the differences in melanization. A comparison of the parental genomes revealed a loss of synteny in the QTL. We found an insertion of a transposable element island of approximately $30 \mathrm{~kb}$, located $1862 \mathrm{bp}$ upstream of the $Z m r 1$ start codon, only in the lighter strain 3D1 (Fig. 2b). The sequences adjacent to the transposable element island, including the full $P k s 1$ gene cluster, showed a high conservation of synteny between the two parental genomes. The transposable element island consisted of 13 transposable elements and possessed both DNA transposons 


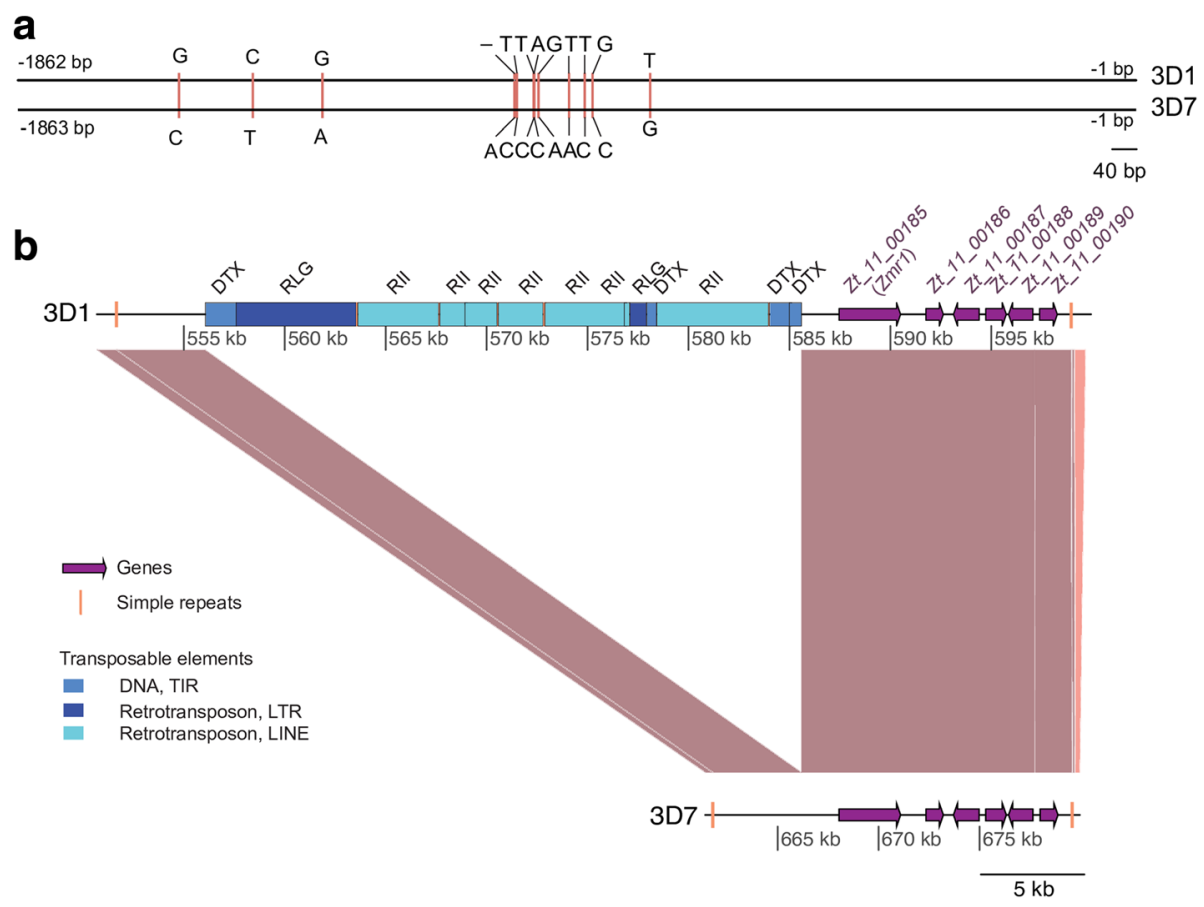

Fig. 2 Differences between 3D1 and 3D7 in Zmr1 regulatory sequences. a Alignment of the nucleotide sequences 1862 bp upstream of the coding sequence of $Z \mathrm{mr} 1$ in 3D1 and 3D7. Vertical pink bars indicate the 12 SNPs in the promoter region. $\mathbf{b}$ Synteny plot of the QTL between 3D1 and 3D7 showing the insertion of an island of transposable elements $1.8 \mathrm{~kb}$ upstream of the start codon of $Z \mathrm{mr} 1$ in the lighter strain 3D1. Brown lines indicate collinear sequences. The positions of genes and transposable elements are shown using purple arrows and blue bars, respectively. Vertical yellow lines indicate simple repeats. The different shades of blue represent different classes of transposable elements that were classified according to the threeletter code described in Wicker et al. [100]. The first letter indicates the class (R=RNA class and D = DNA class), the second letter indicates the order $(L=L T R, I=L i n e, T=T I R)$, and the third letter indicates the superfamily ( $G=$ Gypsy, I = I, X = unknown)

(of the TIR order) and retro-transposons (of the LTR and LINE orders) interspersed by simple repeats (Fig. 2b).

\section{Zmr1 expression is different between the two parental strains}

We hypothesized that changes in non-coding regions could underlie natural variation in levels of melanization. Transposable element insertions upstream of the promoter and/or mutations in the promoter could lead to differential regulation of the genes in the $P k s 1$ gene cluster and, consequently, to different levels of melanin synthesis and accumulation. We found that Zmr1 expression was higher in the darker strain 3D7 than in the lighter strain 3D1 at a time point when differences in melanin accumulation were detected $(7 \mathrm{dpi})$. No significant differences in expression levels were observed at a later developmental stage (9 dpi), when the degree of melanization in 3D1 was higher (Fig. 3, Additional file 4). Thus, we postulated that differential regulation of $Z m r 1$ expression, potentially mediated by differences in non-coding sequences, could underlie differences in this adaptive trait.

\section{Zmr1 regulates melanin biosynthesis in Z. tritici}

To determine the role of $\mathrm{Zmr} 1$ in melanin accumulation in $Z$. tritici, we generated Zmr1 knockout mutants by homologous recombination in strains 3D1 and 3D7 $(\Delta z m r 1)$. No melanin accumulation was observed in $\Delta z m r 1$ mutant colonies grown in vitro or in pycnidia formed on wheat leaves in both genetic backgrounds (Additional file 5), confirming that Zmr1 is required for melanin biosynthesis in $Z$. tritici. We further explored the function of the transcription factor $\mathrm{Zmr} 1$ in regulation of gene expression by pursuing a comparative transcriptomic analysis of the wild-type strains and the $\Delta z m r 1$ mutants, in both 3D1 and 3D7 backgrounds. Twelve genes were downregulated in both $\Delta z m r 1$ mutants (Table 1, Additional file 6). The expression levels of all the genes described to be involved in the DHN melanin biosynthetic pathway were significantly reduced. Remarkably, the expression of Pks1 and Thr1 was nearly abolished in the mutants (Table 1, Additional files 5 and 6). Transcriptomic profiling corroborated the hypothesis that $\mathrm{Zmr} 1$ is a major regulator of the genes involved in the DHN melanin biosynthetic pathway. We showed that DHN melanin is the only type of melanin accumulated in in vitro grown colonies and in $Z$. tritici pycnidia produced in planta. 


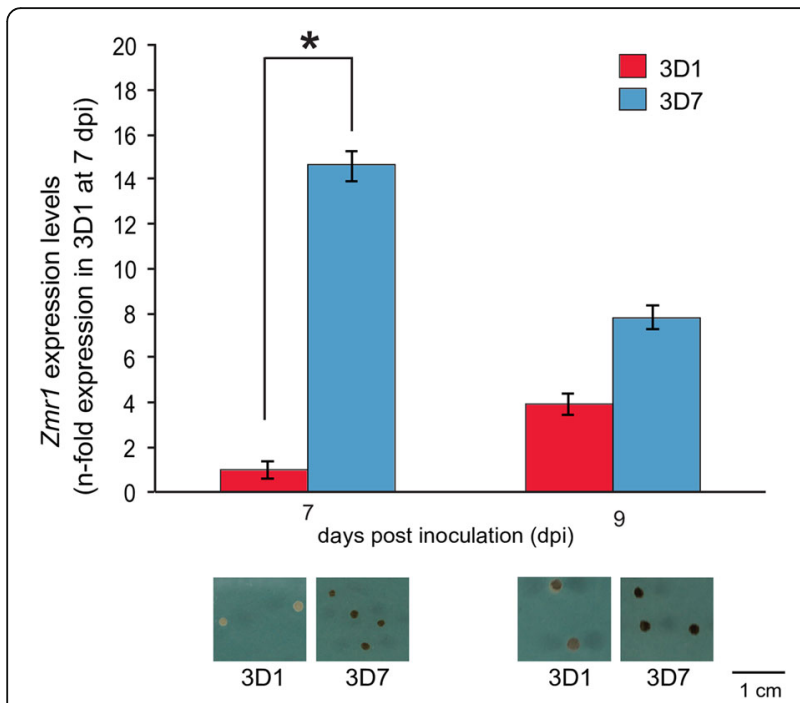

Fig. $3 \mathrm{Zmr1}$ expression is higher in the darker strain (3D7) compared to 3D1. Zmr1 mean expression and standard deviation in 3D1 and 3D7 at 7 and 9 days post inoculation (dpi) relative to the expression of Zmr1 in 3D1 at 7 dpi. Colonies grown on at least three different YMS plates were pooled for RNA extraction. The experiment was performed three times with similar results. Asterisks represent significant differences between 3D1 and 3D7 ( $p$ value $\leq 0.05$, Kruskal-Wallis test). A representative picture of each strain is shown at 7 and $9 \mathrm{dpi}$

$\log _{2}$ fold change ( $\log _{2} \mathrm{FC}$ ) expression values (counts per million mapped) of genes significantly downregulated (Benjamin-Hochberg false discovery rates $(\mathrm{FDR}) \leq$ 0.05 and adjusted $p$ value $\leq 0.05)$ in both $3 \mathrm{D} 1 \Delta z m r 1$ and $3 \mathrm{D} 7 \Delta z m r 1$, compared to their respective wild-type. In bold are genes already described to be involved in the DHN melanin pathway in other fungal species.
Sequence variation in the promoter of $Z m r 1$ contributes to the differential regulation of $Z m r 1$

We next postulated that the basis of the differential accumulation of melanin in 3D1 and 3D7 is the differential expression of Zmr1 (Fig. 3), which could potentially be caused by modifications in the promoter or by the transposable element insertion (Fig. 2). The contribution of promoter modifications to Zmrl expression was evaluated by analyzing allele replacement lines in the 3D7 background. Increased melanization was achieved by in locus expression of both 3D1 and 3D7 Zmr1 alleles in 3D7 $z m r 1$ (3D7 $\left.z z m r 1+Z m r 1_{3 D 1}, 3 D 7 \Delta z m r 1+Z m r 1_{3 D 7}\right)$ compared to the knockout, confirming the role of $Z m r 1$ in melanin biosynthesis in Z. tritici. Remarkably, although the 3D7 allele fully complemented the knockout phenotype, in locus expression of the 3D1 allele led to an intermediate phenotype between the knockout and the wild-type (Fig. 4, Additional file 7), suggesting that differential accumulation of melanin is caused by SNPs in the Zmr1 promoter.

\section{Insertion of a transposable element island upstream of the $Z m r 1$ promoter downregulates $Z m r 1$ expression}

We investigated whether the transposable element insertion in the lighter strain 3D1 modulated Zmr1 expression. We made use of $\Delta z m r 1$ mutants of both 3D1 and 3D7, in which the Zmr1 gene was disrupted by a hygromycin resistance cassette under the control of a constitutive promoter and of the ectopic controls, in which the hygromycin resistance cassette did not disrupt the Zmrl gene but was inserted elsewhere in the genome (Fig. 5a, c). In the 3D7 background, the knockouts and the ectopic lines displayed similar growth in the presence of hygromycin (Fig. 5b). Remarkably, we observed

Table 1 List of genes significantly downregulated in Zmr1 mutants in both 3D1 and 3D7 backgrounds

\begin{tabular}{|c|c|c|c|}
\hline \multirow[t]{2}{*}{ Gene ID } & \multicolumn{2}{|l|}{$\log _{2} F C$} & \multirow[t]{2}{*}{ Annotation } \\
\hline & 3D1 & $3 \mathrm{D} 7$ & \\
\hline Zt09_11_00186 & -9.92 & -8.93 & 1,3,8-trihydroxynaphthalene reductase $(T h r 1)$ \\
\hline Zt09_4_00395 & -5.35 & -6.48 & Similar to cytochrome p450 \\
\hline Zt09_2_00626 & -3.66 & -1.61 & Hypothetical protein \\
\hline Zt09_11_00184 & -3.22 & -4.35 & Polyketide synthase (Pks1) \\
\hline Zt09_2_00074 & -2.5 & -5.09 & Predicted protein \\
\hline Zt09_3_00872 & -2.2 & -1.76 & Homolog of Aspergillus yellowish green (Ayg1) \\
\hline Zt09_4_00393 & -2.19 & -3.27 & Hypothetical protein \\
\hline Zt09_1_00117 & -2.15 & -4.14 & Hypothetical protein \\
\hline Zt09_4_00394 & -2.15 & -2.4 & Salicylate hydroxylase \\
\hline Zt09_3_00063 & -2.01 & -5.28 & Glycoside hydrolase family 18 protein \\
\hline Zt09_11_00185 & -1.76 & -1.94 & Zymoseptoria melanin regulation $1(\mathrm{Zmr} 1)$ \\
\hline Zt09_1_00074 & -1.76 & -3.82 & Hypothetical protein \\
\hline Zt09_1_00268 & -1.67 & -2.5 & Scytalone dehydratase $(S c d 1)$ \\
\hline
\end{tabular}




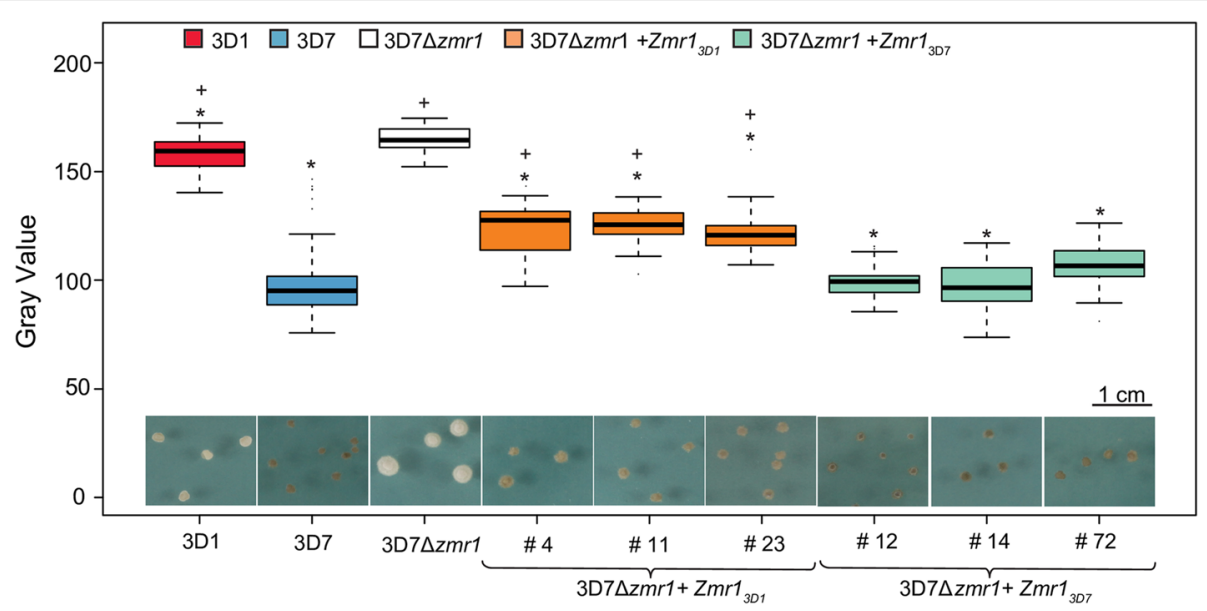

Fig. 4 Sequence variation in the Zmr1 promoter contributes to differences in melanin accumulation. Gray values for 3D1, 3D7, the Zmrl knockout

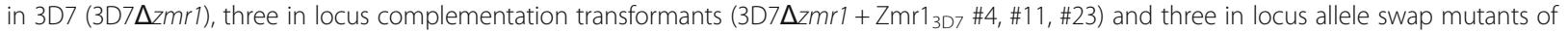
$Z m r 1\left(3 D 7 \Delta z m r 1+Z m r 1_{3 D 1} \# 12\right.$, \#14, \#72), all grown for 7 days. Asterisks $\left(^{*}\right)$ and plus (+) indicate significant differences in gray values of each strain with respect to the gray value of $3 D 7 \Delta z m r 1$ and $3 D 7$, respectively ( $p$ value $\leq 0.05$, Kruskal-Wallis). At least 20 colonies (replicates) grown on three different plates were evaluated. The experiment was performed twice with similar results

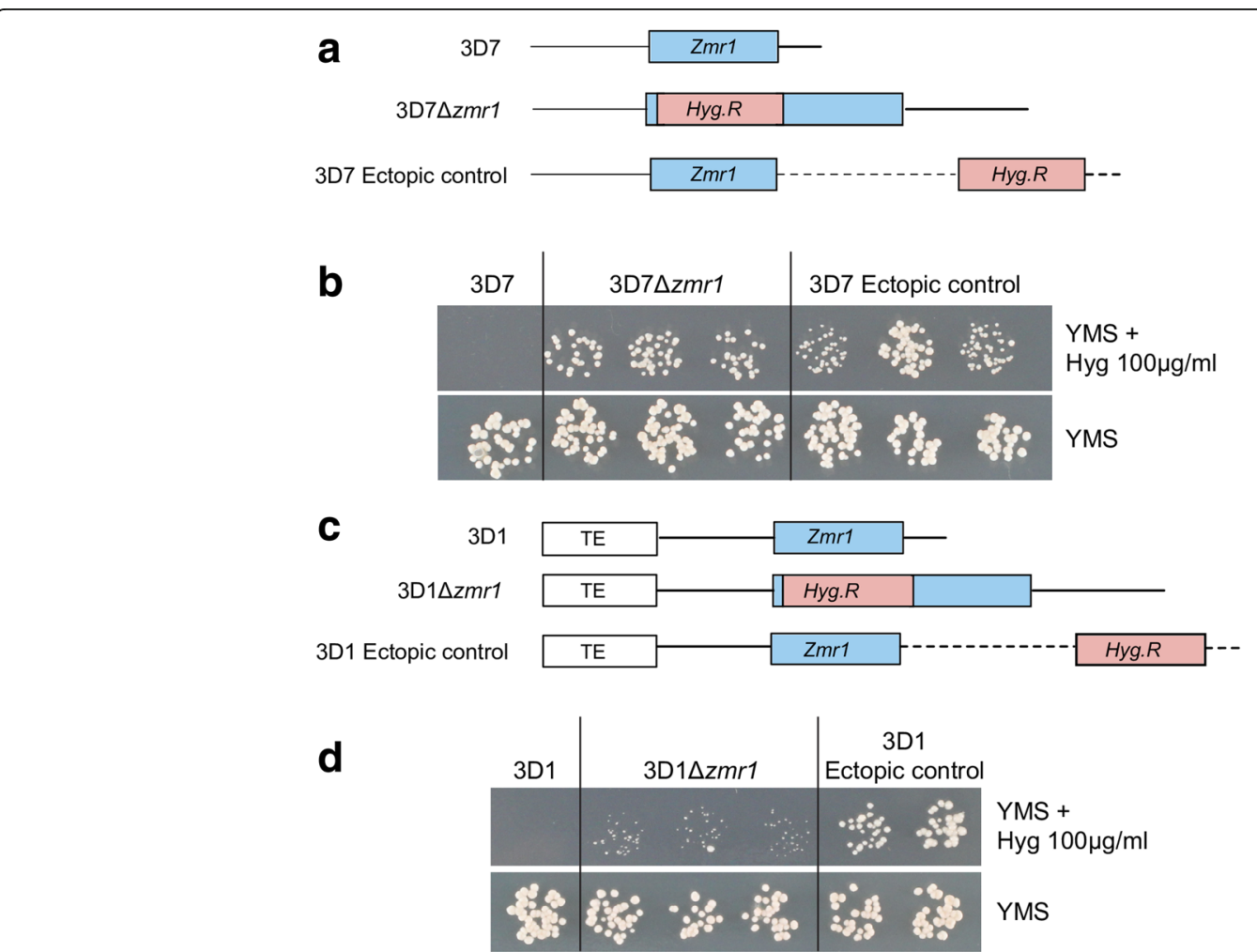

Fig. 5 The transposable element insertion upstream of $Z m r 1$ influences the expression of adjacent genes. a Schematic representation of the $Z m r 1$ locus in the wild-type 3D7 and the insertion of a hygromycin resistance cassette (Hyg.R) in 3D7Dzmr1 and in the ectopic controls. b Growth of 3D7, 3D7 $\Delta z \mathrm{mr}$, and the ectopic controls in yeast malt sucrose (YMS) plates with and without hygromycin $(100 \mu \mathrm{g} / \mathrm{ml})$. c Schematic representation of the $Z m r 1$ locus in the wild-type 3D1 and the insertion of a hygromycin resistance cassette (Hyg.R) in 3D1 $\Delta z m r 1$ and in the ectopic controls. d Growth of 3D1 $\Delta \mathrm{zmr} 1$ was reduced compared to growth of the ectopic controls in the presence of hygromycin $(100 \mu \mathrm{gg} / \mathrm{ml})$. Growth is normal for all lines in the absence of hygromycin. The experiment was performed three times with similar results 
that growth of all three independent $\Delta z m r 1$ knockouts in the 3D1 background was lower than growth of the ectopic transformants in hygromycin-containing medium (Fig. 5d). Hence, we hypothesized that the transposable element cluster silenced the expression of the hygromycin resistance gene, with the observed phenotype in the mutant likely reflecting the contribution of the transposable element insertion to Zmr1 expression regulation.

To confirm the role of transposable elements in downregulating $\mathrm{Zmr} 1$ expression, attempts were made to generate in locus complementation or allele replacement transformant lines of 3D1 $1 \Delta m r 1$. However, no successful transformants were obtained. Instead, we replaced the entire transposable element island $(30 \mathrm{~kb})$ with a hygromycin resistance cassette. Three independent knockout lines $(\triangle T E)$ of the transposable element insertions were obtained and analyzed for melanin accumulation in vitro at $7 \mathrm{dpi}$. The transposable element deletion mutants were much darker than the wild-type 3D1 (Fig. 6a, Additional file 8). Furthermore, expression levels of $Z m r 1$ in the transposable element knockouts were significantly higher than those in the wild-type 3D1 (Fig. 6b). Overall, these results demonstrate that the transposable element island upstream of $Z m r 1$ in the less melanized strain negatively regulates gene expression and contributes to the variability in melanin accumulation between the two strains.

\section{Melanin lowers fungicide sensitivity but has an associated fitness cost}

We observed that the non-melanized mutants grew faster than the corresponding wild-types (Fig. 7a, b, Additional files 9 and 10). The growth rates of melanized 3D1 and 3D7 were, respectively, 61 and 34\% lower than those in non-melanized mutants, suggesting that melanin production has a fitness cost for Z. tritici. We aimed to explore possible biological roles for melanin in
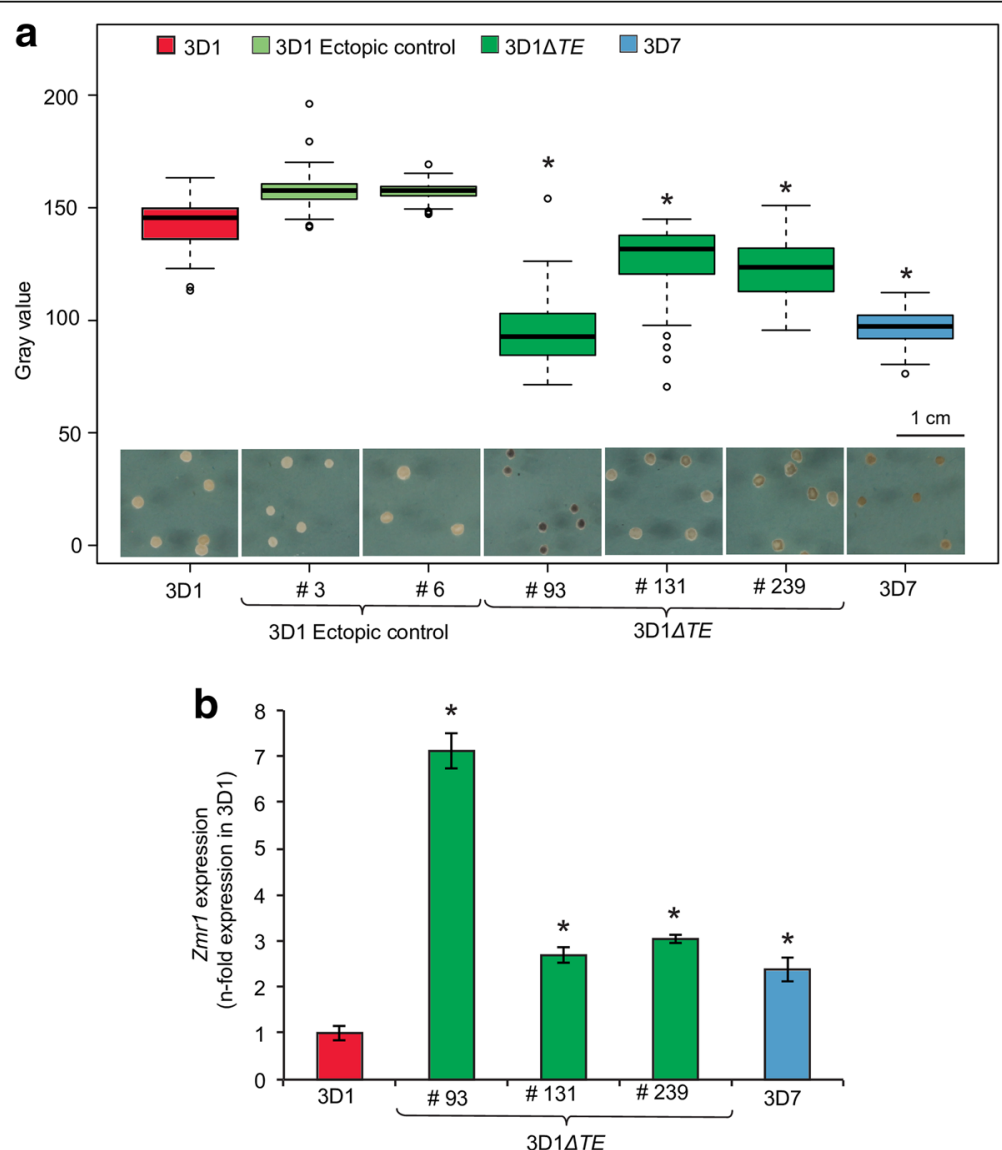

Fig. 6 The transposable element insertion in the lighter strain downregulates $Z m r 1$ expression. a Deletion of the transposable element island leads to significantly more melanin accumulation in 3D1 at 7 days post inoculation (dpi). Distribution of gray values for at least 35 colonies at $7 \mathrm{dpi}$ for 3D1, 3D7, the transposable element deletion mutants in the 3D1 background (3D1 $\triangle T E \# 93, \# 131$ and \#239), and the ectopic controls (\#3 and \#6). Asterisks indicate significant differences in gray values with respect to the wild-type 3D1 ( $p$ value $\leq 0.05$, Kruskal-Wallis). The experiment was performed three times with 3D1 $\Delta \mathrm{TE} \# 93$ and twice with \#131 and \#239 and we obtained similar results. $\mathbf{b}$ Zmr1 expression levels in the transposable element knockouts (\#93,\#131, \#239) are significantly higher than those in the wild-type 3D1 at $7 \mathrm{dpi}$. $Z \mathrm{mr} 1$ expression values are relative to the expression of $Z m r 1$ in $3 \mathrm{D} 1$. Means and standard deviations of three technical replicates are shown. Asterisks $\left(^{*}\right)$ represent statistical differences with the wild-type ( $p$ value $\leq 0.05$, Kruskal-Wallis test). The experiment was performed twice and we obtained similar results 

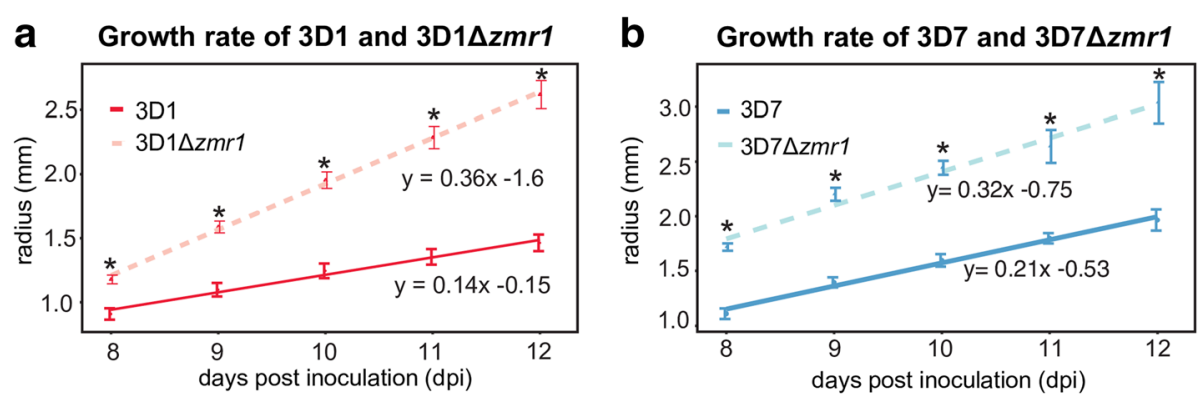

C Sensitivity of 3D7 $\Delta z m r 1$ and 3D7 to bixafen

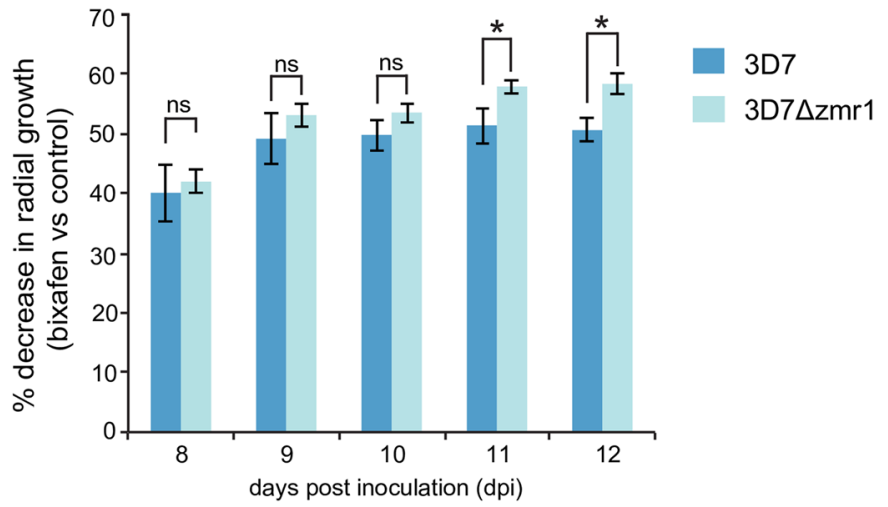

Fig. 7 Melanin accumulation is associated with reduced growth and resistance to SDHI fungicides. $\mathbf{a}$, $\mathbf{b}$ Colony radius over time of melanindeficient mutants in 3D1 (a) and 3D7 (b) backgrounds. The experiment was performed three times with similar results. c Melanin protects Z. tritici against the SDHI fungicide bixafen. Percentage decrease in growth of the wild-type 3D7 and the 3D7 $\Delta z m r 1$ knockout in the presence and absence of the fungicide at each time point (8-12 dpi). Mean and standard error of differential radial size of colonies grown on three independent plates are presented. The experiment was performed twice with similar results. Asterisks $\left(^{*}\right)$ indicate statistical differences between wild-type and knockout at each time point ( $p$ value $\leq 0.05$, Kruskal-Wallis). ns non-significant

Z. tritici. Virulence of a non-melanized mutant was not altered compared to the wild-type strain after 21 days of infection in wheat plants (Additional files 11 and 12). Furthermore, pycnidiospores produced under controlled greenhouse conditions in the albino pycnidia of $\Delta z m r 1$ were fully viable. Thus, we found no evidence that melanin plays a role in host colonization or pathogen reproduction. To evaluate the role of melanin in fungicide sensitivity, we grew the wild-type 3D7 and the non-melanized mutant 3D7 $\Delta z m r 1$ colonies in rich media until 3D7 was melanized ( $5 \mathrm{dpi}$ ) and then we treated the colonies with the succinate dehydrogenase inhibitor (SDHI) fungicide bixafen. The decrease in growth in the presence of the fungicide of the non-melanized mutant $3 \mathrm{D} 7 \Delta z m r 1$ was higher than that of the wild-type 3D7, indicating that melanin lowers the sensitivity of $Z$. tritici to bixafen (Fig. 7c, Additional file 13). However, the non-melanized mutant was not more sensitive to the azole fungicide propiconazole than the wild-type (Additional file 13). These data demonstrate that melanin can specifically protect $Z$. tritici against SDHI fungicides, but its production has a negative effect on growth. We propose that modulation of Zmr1 expression potentially balances its beneficial functions against the growth costs associated with melanin synthesis.

\section{Transposable element insertions regulate $Z m r 1$ expression} and melanin accumulation in $Z$. tritici populations

We hypothesized that transposable element insertions similar to those in 3D1 could contribute to differences in melanization at the species level. We analyzed the presence of transposable elements upstream of $Z m r 1$ in the reference strain IPO323 [44]. Zmr1 had the same protein sequence as 3D1 and 3D7 and, as expected, melanization levels of IPO323 increased with age (Additional file 14A). No transposable element was identified in the immediate vicinity of $Z m r 1$ in IPO323 and the closest transposable element insertion was located $135 \mathrm{~kb}$ upstream of the start codon of Zmrl (Additional file 14). We used Illumina reads from $132 \mathrm{Z}$. tritici strains from four different global field populations and screened for mutations in the upstream promoter region and the presence of transposable elements upstream of the $Z m r 1$ gene. The amino acid sequence of Zmr1 was highly conserved in all the strains, with an 
average identity of $99 \%$. Sixty-five percent of the sites in the region upstream of the coding sequence of $\mathrm{Zmrl}$ were polymorphic. Of the $12 \mathrm{SNPs}$ in the Zmr1 promoter in the lighter 3D1 strain, eight were also present in the other analyzed strains of $Z$. tritici (Additional file 15) and could potentially contribute to variation in melanization levels. Twelve of the strains (including 3D1) had at least one transposable element insertion within $4 \mathrm{~kb}$ upstream of the $Z m r 1$ gene. In two additional strains, short scaffold lengths prevented a full screening for the presence of transposable elements. It is likely that all of the identified insertions were the consequence of independent insertion events because they consisted of different types of transposable elements (including a retrotransposon, six DNA transposons, and three unclassified transposable elements) and were located at different positions upstream of Zmrl (Fig. 8). We selected 11 strains with transposable element insertions and 22 without any insertion upstream of $Z m r 1$ to evaluate the effects of the transposable elements on the regulation of melanin production. Melanin accumulation among these strains was highly variable, with gray values ranging from 91 to 161 at $7 \mathrm{dpi}$ (Fig. 9, Additional files 16 and 17). Insertions of transposable elements had a significant negative effect on melanin accumulation
(Fig. 8b, Additional file 16). Furthermore, transposable element insertions negatively affected $Z m r 1$ expression levels (Fig. 8c). These results further support the hypothesis that the transposable element insertion polymorphism affects Zmrl expression and contributes to the observed phenotypic diversity for melanin accumulation in Z. tritici.

\section{Discussion}

Melanin is thought to play an important role in adaptation to changing environments for many fungi. Given its importance, evolution is likely to favor the emergence of genetic mechanisms that enable a variable regulation of melanin accumulation that can balance fitness costs associated with melanin synthesis against the survival advantage that may be gained under hazardous conditions. Here, we demonstrated that differences in regulation of expression of the gene encoding the transcription factor Zmr1 can be governed by both transposable elements and variation in promoter sequences, and contribute to variation in melanization levels.

Alterations in regulatory pathways are known to contribute to natural variation in complex traits and differential regulation of gene expression has long been associated with morphological differences among
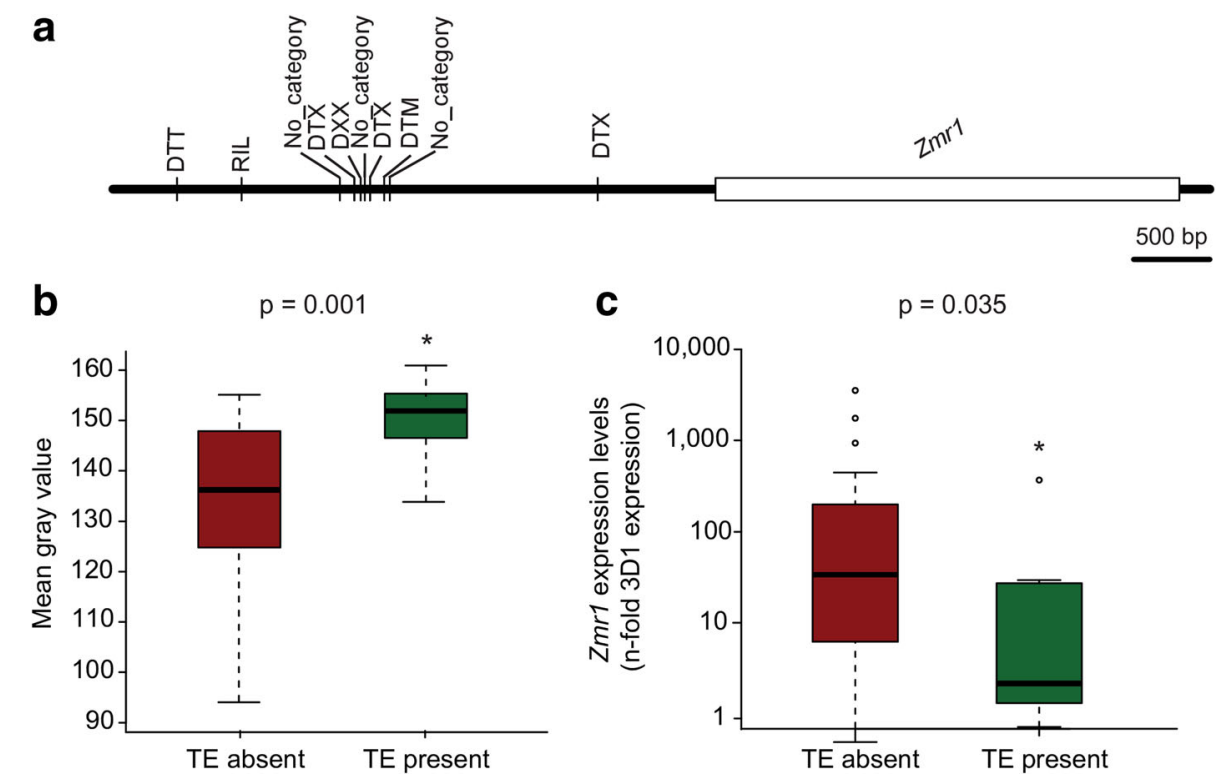

Fig. 8 Transposable element insertions regulate Zmrl expression and melanin accumulation in Z. tritici populations. a Schematic representation of the location and classification of the transposable element insertions upstream of $Z m r 1$ in different $Z$. tritici strains from a worldwide collection. The transposable elements were classified according to the three-letter code described in Wicker et al. (2007) [100]: The first letter indicates the class $(R=$ RNA class and $D=$ DNA class); the second letter indicates the order $(I=L i n e, T=T I R, X=$ unknown); and the third letter indicates the superfamily $(L=L 1, M=$ Mutator, $T=T C 1$-Mariner, $X=$ unknown). $\mathbf{b}$ Transposable element insertions upstream of Zmr1 significantly contribute to a reduction in melanin accumulation, according to Kruskal-Wallis ( $p$ value $=0.0008$, indicated with asterisks). Gray value distributions of $Z$. tritici strains with and without transposable element insertions upstream of Zmr1. The experiment was performed three times and we obtained similar results. c Transposable element insertions upstream of $Z m r 1$ negatively affect $Z m r 1$ expression (Kruskal-Wallis, $p$ value $=0.035$, indicated with asterisks). Distribution of the mean expression of Zmrl (relative to 3D1 at 7 days post inoculation) in each Z. tritici strain with and without transposable element insertions upstream of Zmr1. The experiment was performed twice with similar results 


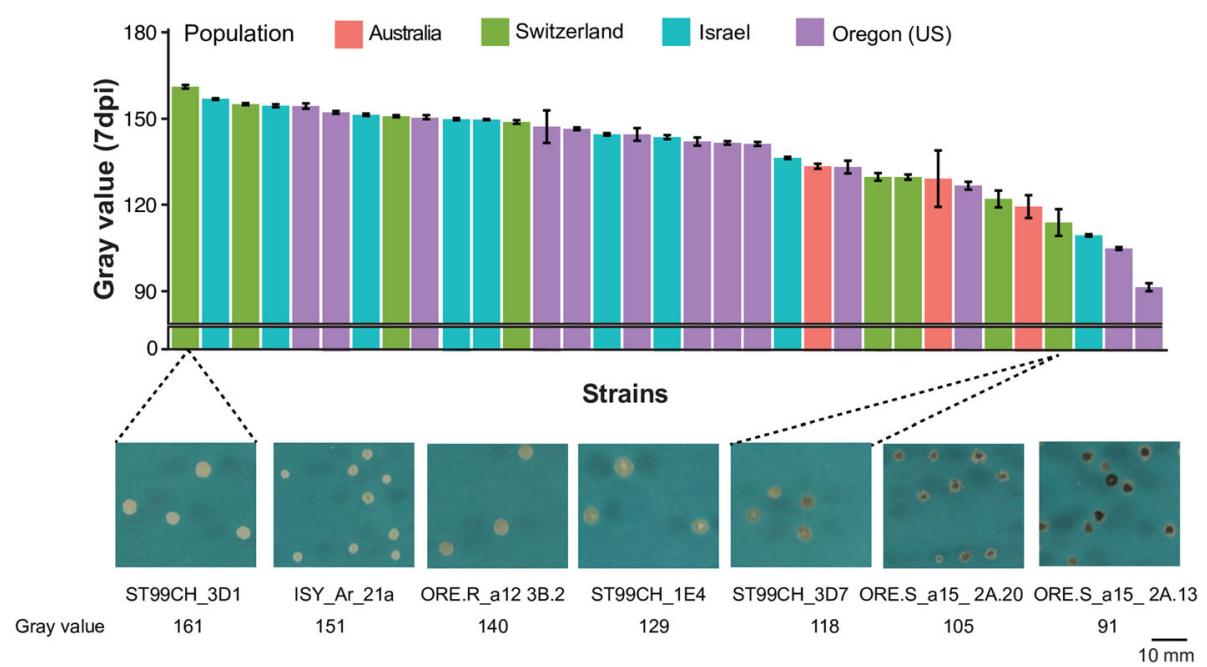

Fig. 9 High diversity in melanin levels is exhibited among strains from four worldwide populations. Gray values of 37 different $Z$. tritici strains from four different field populations across the world. The colors of the bars indicate the population to which the strains belong. Means and standard errors of the gray values were based on colonies grown for 7 days. Some examples of $Z$. tritici strains illustrating the diversity in melanin accumulation are shown in the lower panel. The experiment was performed three times with similar results

individuals within plant and animal species. For example, selection by maize breeders for a reduction in branching most probably targeted regulatory differences of the gene encoding the transcription factor Teosinte Branched 1, which represses the growth of axillary meristems [45]. In stickleback fish, the differential expression of a bone morphogenetic protein due to a transposon insertion led to changes in the size of armor plates involved in defense [46]. The diversity of wing pigmentation patterns found in fruit fly species is acquired through regulatory changes affecting enzymes involved in pigment deposition $[47,48]$. These examples illustrate well the effects of regulatory pathways on complex traits in model plant and animal species. Although several studies suggested that regulatory changes could also play a role in phenotypic variation in fungi, these studies were limited mainly to yeast [49-54]. Much less is known about the importance of regulatory mutations for maintaining phenotypic variation in filamentous fungi $[17,18]$. Here we demonstrated a significant effect of variation in cis-regulatory elements on a complex trait in a fungal plant pathogen. Modification of the regulatory sequences of $Z m r 1$ had a major effect on the accumulation of melanin in Z. tritici. Our findings indicate that regulatory modifications can play a major role in fungal adaptation to variable environments. In several recent population genomic analyses of fungi, SNPs significantly associated with a phenotypic trait were identified in non-coding regions of the genome [36, 55]. We hypothesize that these SNPs are involved in modification of regulatory pathways that subsequently lead to the observed phenotypic variation.

\section{Diversity in melanin levels and its role in adaptation}

Melanin is a widely distributed compound in eukaryotes that can affect fitness. The biological functions of melanin differ substantially among species [31, 43, 56, 57]. In plant pathogens, such as Pyricularia grisea, Colletotrichum lindemuthianum, and Colletotrichum lagenarium, melanin accumulation in the appressorium is essential for direct penetration of the host epidermis [42, 58]. Because Z. tritici enters the host through the stomata, melanin is not required to initiate infection. The lack of significant differences in virulence between isogenic melanized and non-melanized strains of $Z$. tritici suggests that melanin does not play a major role in colonization under the tested conditions. However, we cannot discount a virulence function for melanin under natural conditions, where variation in UV radiation, host genotypes, and interactions with other microbes are likely to play important roles. $Z$. tritici pycnidia are highly melanized, and melanin likely protects the embedded pycnidiospores. In other organisms, melanin shields against stress [43] and the degree of melanization can be correlated with the degree of resistance to stress [59]. We found that melanin can lower sensitivity to an SDHI fungicide (Additional file 13), suggesting that frequent applications of SDHI fungicides onto wheat fields may select for strains that can accumulate higher levels of melanin. The capacity of melanin to shield against toxic compounds could reflect a role for melanin in protection against antimicrobials produced under natural conditions by microbial competitors or by the host $[43,60,61]$. The melanin protection we observed against fungicides was specific. The inability of melanin to protect against azoles might be related to 
the fact that azoles do not bind to melanin, so azoles can reach their target site even in melanized cells [62]. Though melanin can contribute to survival in fluctuating environments, our experiments indicated that melanin production has a fitness cost that results in reduced growth. We found that $Z$. tritici strains exhibit temporal differences in melanin accumulation. We postulate that these differences reflect selection operating to balance rates of growth with survival to environmental stress. Under this scenario, melanin accumulation illustrates how a trade-off between adaptation and growth can contribute to variation in a trait.

\section{Variability in melanin accumulation is caused by differential regulation of gene expression}

Our approach revealed that variability in melanin accumulation is mediated by differential regulation of expression of the Zmr1 gene. Zmr1 encodes a transcription factor that regulates expression levels of genes in the melanin biosynthetic cluster. We characterized two regulatory layers mediating variation in $Z m r 1$ expression: promoter sequence modifications and an insertion of transposable elements upstream of the promoter. Twelve SNPs in the promoter of Zmrl underlie differential regulation of melanin accumulation in the light and dark strains. Although the individual effects of these 12 mutations have not yet been tested, we hypothesize that at least one of these promoter mutations alters the levels of $Z m r 1$ transcription.

An island of 13 transposable elements of approximately $30 \mathrm{~kb}$ is located upstream of the $Z m r 1$ promoter in the lighter strain and delays Zmr1 expression. We demonstrated the contribution of the transposable elements in downregulating melanin accumulation by removing the entire transposable element island, which led to an increase in Zmrl expression and melanin accumulation. The transposable element-mediated downregulation of $Z m r 1$ is transient, as the differences in $Z m r 1$ expression between the lighter and darker strain decrease with age. The transposable element island hinders $Z m r 1$ expression either by blocking the activity of activators upstream of the transposable elements or by epigenetically silencing adjacent regions. Remarkably, we observed a silencing effect of the hygromycin resistance gene under the control of a constitutive promoter when it was located at the Zmr1 locus, downstream of the transposable element island in the 3D1 strain. The expression of the hygromycin resistance gene was higher when it was located ectopically or at the $Z m r 1$ locus in the 3D7 background. These findings suggest that the transposable element insertions reduce the expression of Zmrl in the lighter strain through epigenetic mechanisms.
Transposable elements are frequently associated with heterochromatic regions of the genome and this limits transposable element activity and transcription [40, 63-67]. The spread of the heterochromatic state of the transposable elements to neighboring genes silences their expression, as shown in other organisms [15, 68-71]. Frequently, under stressful conditions, some families of transposable elements are transcriptionally activated [72-74]. This suggests that transposable elements may provide a mechanism to specifically regulate expression of nearby genes under stressful conditions [21, 75, 76]. In Epichloë festucae two genes involved in the synthesis of alkaloids are located in a transposable element-rich region and are epigenetically silenced in axenic culture. Epigenetic silencing and de-silencing were shown to provide an important regulatory layer to specifically produce the alkaloids during host colonization [63]. In the pathogenic fungus Leptosphaeria maculans, effector genes are located in heterochromatic regions rich in transposable elements. Insertions of transposable elements were shown to modify the epigenetic state of nearby effector genes and consequently modulate their expression patterns [77]. In maize, insertion of a transposable element and the resulting spread of DNA and histone methylation marks to the cis-regulatory region of a gene reduces the accessibility for transcription factors and the RNA polymerase, thus altering expression levels upon attack by Fusarium graminearum [15]. We postulate that regulation of Zmr1 by insertions of transposable elements is mediated by similar mechanisms, which involves the spreading of epigenetic marks to Zmrl in the lighter strain. In this way, transposable element insertions can provide a new layer of gene regulation that can optimize fitness in fluctuating environments.

\section{Genomic rearrangements modulate melanin levels in $Z$. tritici populations}

Two antagonistic consequences of melanin accumulation, protection from stress and decrease in growth rate, suggest the need for variable regulation of melanin synthesis to survive in different environments. During host colonization, $Z$. tritici is exposed to different micro-climatic conditions and is subjected to environmental changes, depending on its spatial location during host colonization [25, 78]. It is likely that this spatial and temporal environmental heterogeneity leads to diversification of melanization levels in Z $Z$. tritici. Fluctuations in macro-climate may also select for diversification in melanization, with episodes of severe heat, cold, drought, or UV radiation likely favoring strains with higher melanization, while less melanized strains may have higher fitness during less stressful weather conditions. The 
significant variability in the degree of melanization exhibited among different strains of $Z$. tritici can have many underlying causes, but we hypothesize that most of these differences reflect local adaptation.

The genome of $Z$. tritici contains approximately $17 \%$ repetitive elements $[39,69]$. Transposable element insertions can cause adaptive variation and contribute to pathogen evolution. Transposable elements are frequently associated with stress-related genes and are considered to contribute to their diversification $[22,23,76$, 79] but how transposable elements drive adaptation remains to be fully understood. Here we show that transposable elements contribute to phenotypic diversity by regulating gene expression. Independent insertions of transposable elements in $Z$. tritici contributed to differential regulation of $Z m r 1$ expression and led to diversification of melanin accumulation.

\section{Conclusions}

We demonstrated that diversity in melanin accumulation is determined by differential regulation of gene expression instead of through mutations in coding sequences. Both single nucleotide polymorphisms in the promoter region of the $Z m r 1$ gene and transposable element insertions altered the accumulation of melanin. The complexity at the locus suggests that a sophisticated regulatory mechanism has evolved to balance the trade-offs between growth and melanin production. We believe that variation in transposable element insertions creates differential regulatory patterns through chromatin modification, generating new epialleles. We elucidated how transposable elements can facilitate the diversification of adaptive traits by generating regulatory variation that can regulate fitness-relevant gene expression.

\section{Methods}

Growth conditions for $Z$. tritici strains and bacterial strains All the $Z$. tritici strains analyzed in this study, except for IPO323, were previously collected from wheat fields in four different locations: Australia, Israel, Switzerland, and Oregon, USA, during 1990-2001 [36, 80, 81]. The reference strain IPO323 was kindly provided by Gert H.J. Kema (Wageningen University, The Netherlands). The Z. tritici Swiss strains ST99CH_3D1 (abbreviated as 3D1) and ST99CH_3D7 (abbreviated as 3D7) collected in 1999 [33, 82] were used for genetic modifications. Wild-type and genetically modified $Z$. tritici strains were grown in $50 \mathrm{ml}$ of yeast sucrose broth (YSB, 1\% w/v yeast extract, $1 \% \mathrm{w} / v$ sucrose) with $50 \mu \mathrm{g} / \mathrm{ml}$ kanamycin sulfate in 100-ml Erlenmeyer flasks at $18{ }^{\circ} \mathrm{C}, 120 \mathrm{rpm}$ for 6 days. Blastospores from the wild-type and genetically modified $Z$. tritici strains were collected after 6 days of growth in YSB. Liquid cultures were filtered through double-layered sterile cheesecloth and blastospores were collected by centrifugation $\left(3273 g, 15 \mathrm{~min}, 4{ }^{\circ} \mathrm{C}\right)$. The supernatant was discarded; blastospores were washed twice and re-suspended in sterile deionized water and stored on ice until use ( $0-1$ day). The concentrations of the spore suspensions were determined using $\mathrm{KOVA}^{\circ}$ Glasstic $^{\circ}$ counting chambers (Hycor Biomedical, Inc., USA). Yeast malt sucrose agar (YMS, $0.4 \% w / v$ yeast extract, $0.4 \% w / v$ malt extract, $0.4 \% w / v$ sucrose, $1.5 \% w / v$ agar) and potato dextrose agar (PDA) were used for growing $Z$. tritici strains on Petri plates.

Escherichia coli strains $\mathrm{NEB}^{\circ}$ 5-alpha (New England Biolabs) or HST08 (Takara Bio, USA) were used for molecular cloning. E. coli strains were grown on DYT media $(1.6 \% w / v$ tryptone, $1 \% w / v$ yeast extract, $0.5 \% \mathrm{NaCl})$ amended with kanamycin sulfate $(50 \mu \mathrm{g} / \mathrm{ml})$ at $37{ }^{\circ} \mathrm{C}$. Agrobacterium tumefaciens strain AGL1 was used for $A$. tumefaciens-mediated transformation of $Z$. tritici. A. tumefaciens was grown in DYT media containing kanamycin sulfate $(50 \mu \mathrm{g} / \mathrm{ml})$, carbenicillin $(100 \mu \mathrm{g} / \mathrm{ml})$, and rifampicin $(50 \mu \mathrm{g} / \mathrm{ml})$ at $28{ }^{\circ} \mathrm{C}$, unless stated otherwise.

\section{QTL mapping}

Phenotypic data (gray values of the mapping population at $8 \mathrm{dpi}$ ) and restriction site-associated DNA sequencing (RADseq) data from the progeny of the cross between 3D1 and 3D7 described earlier [33] were used for QTL mapping, using the same protocol described in Meile et al. [83]. QTL re-mapping of chromosome 11 alone was performed in R/qtl version v1.40-8 [84] by simple interval mapping (SIM) analysis as described previously [33].

\section{Melanization analysis}

The degree of melanization in each $Z$. tritici strain was estimated by plating approximately 100 blastospores on YMS plates. Plates were then randomized and incubated in the dark at $22{ }^{\circ} \mathrm{C}$ and $70 \%$ humidity. Digital images of the plates were taken through the Petri plate lid at different time points, using standardized settings [33]. Gray value, a proxy for degree of melanization, was estimated for each colony using ImageJ [85]. The gray scale ranges from 0 to 255, where 0 represents the darkest shade of black and 255 represents the lightest shade of white. Gray value of colonies grown on at least three independent Petri plates was measured.

\section{Measurements of growth rate and fungicide sensitivity assays}

Because the 3D7 $\Delta z m r 1$ mutant grew as hyphae instead of as blastospores in YMS (Additional file 18), it was not possible to make a proper evaluation of its growth rate on YMS. Thus, we performed these experiments on PDA, on which both knockouts grew with a morphology that was similar to the wild-type strains (Additional file 18). Colony 
size was evaluated as previously described at 7-12 dpi [30]. The growth curve for wild-type strains and knockouts was obtained by plotting radial growth (millimeter) over time and fitted to a linear model (Pearson's correlation coefficient value $\left(r^{2}\right.$ value $\left.\left.>0.9\right)\right)$. Growth rate $(\mathrm{mm} /$ day $)$ was estimated by calculating the slope of the growth curve. Analysis of covariance (ANCOVA) was performed to determine if there were significant differences in growth rate ( $p$ value $\leq 0.05)$. Significant differences in colony size at each time point (Kruskal-Wallis, $\leq 0.05$ ) were evaluated between $\Delta z m r 1$ and the wild-type. The experiment was performed three times. To perform fungicide sensitivity assays comparing the wild-type 3D7 and non-melanized 3D7 $\Delta z m r 1$ line, a 100-blastospore suspension was plated on sterile Whatman filter paper, grade 1 (Huber lab), placed on PDA plates. Three plates per strain and condition were incubated in the dark at $22{ }^{\circ} \mathrm{C}$ with $70 \%$ humidity. After 5 days, the plates were photographed and the filter papers were transferred to PDA plates supplemented with fungicides $(0.75 \mathrm{ppm}$ of bixafen or $0.75 \mathrm{ppm}$ of propiconazole, Syngenta, Basel, Switzerland) or control PDA plates without any fungicides. The strains were grown under the same conditions as before and digital images were captured every $24 \mathrm{~h}$ until they were 12 days old. The radial growth rates were calculated as described earlier using ImageJ [41]. The percentage decrease in colony radius in the presence of each fungicide was calculated at each time point. The experiment was performed twice.

\section{Generation of Z. tritici transformants}

All the amplifications were performed using Phusion high-fidelity DNA polymerase from NEB (Ipswich, MA, USA). Zmr1 disruptant mutants in both 3D1 and 3D7 backgrounds were generated by inserting a hygromycin resistance cassette into the $Z m r 1$ gene 13 base pairs (bp) after the start codon using homologous recombination (Additional file 19). Up-flanking and down-flanking regions (approximately $1000 \mathrm{bp}$ ) of the site of integration were PCR-amplified from either 3D1 or 3D7 genomic DNA. A hygromycin resistance cassette with the desired overlap for In-Fusion cloning was amplified from the plasmid pES6 (obtained from Eva Stukenbrock, Kiel University). The flanking regions and the hygromycin resistance cassette were fused to the binary vector backbone of pES1 (obtained from Eva Stukenbrock, Kiel University) in their respective order (Additional file 19) by a single-step In-Fusion reaction (Takara Bio, Mountain View, CA, USA) following the manufacturer's instructions and then cloned in E. coli.

Constructs to generate knockouts of the transposable elements in the 3D1 background were obtained in a similar way, except that these mutants were generated by replacing the transposable elements by the hygromycin resistance cassette (Additional file 19).
For generating in locus allele swaps and complementation lines, the full-length $\mathrm{Zmrl}$ gene along with 1863 and 1862 bp upstream of the start codon in 3D7 and $3 \mathrm{D} 1$, respectively, and 539 bp downstream of the stop codons were amplified and fused to a geneticin resistance cassette amplified from the pCGEN vector [86] and the vector backbone of pES1 as described earlier (Additional files 19 and 20). This intermediate construct was used to amplify the full $\mathrm{Zmrl}$ gene fused to the geneticin resistance cassette. Additionally, approximately $1 \mathrm{~kb}$ upstream and downstream of the insertion site in 3D7 were amplified and the three amplicons were fused to the binary vector backbone of pES1 as described earlier (Additional files 19 and 20).

Mutation-free plasmids were transformed into the $A$. tumefaciens strain AGL1 [87] by electroporation and screened on DYT medium supplemented with $50 \mu \mathrm{g} / \mathrm{ml}$ rifampicin, $50 \mu \mathrm{g} / \mathrm{ml}$ carbenicillin, and $40 \mu \mathrm{g} / \mathrm{ml}$ kanamycin at $28^{\circ} \mathrm{C}$. A tumefaciens-mediated transformation of $Z$. tritici was performed as previously described [83, 88, 89]. Selection of transformants was performed on YMS plates containing $200 \mu \mathrm{g} / \mathrm{ml}$ cefotaxime and the corresponding antibiotic, either hygromycin at $100 \mu \mathrm{g} / \mathrm{ml}$ (Neofroxx, Germany) or geneticin at $150 \mu \mathrm{g} / \mathrm{ml}$ (Thermo Fisher Scientific) at $18^{\circ} \mathrm{C}$ for $8-12$ days. Individual colonies were then streaked onto YMS plates containing the corresponding antibiotic and grown at $18{ }^{\circ} \mathrm{C}$ for 1 week. After one round of selection, single colonies were transferred to YMS plates without a selection agent and transformants were screened for the correct inserts by colony PCR using KAPA3G Plant DNA polymerase (Kapa Biosystem, Massachusetts, USA) and specific primers (Additional file 20). These amplicons were further sequenced (Microsynth AG, Balgach, Switzerland) to confirm the correct integration. The copy number of the transformants was determined by performing quantitative PCR (qPCR) on DNA isolated from transformed Z. tritici strains using Qiagen plant DNeasy kit (Qiagen) and specific primers for the antibiotic resistance marker and for the housekeeping genes TFIIIC1 or 18s rRNA (Additional file 20), as previously described [83]. DNA from wild-type $Z$. tritici strains without the transgene, DNA from $Z$. tritici strains harboring a single transgene, and negative water controls were included in all analyses.

\section{Hygromycin resistance assay}

To test the sensitivity to hygromycin of 3D1 $1 \Delta m r 1$ and $3 \mathrm{D} 7 \Delta z m r 1$, their respective wild-types, and the ectopic controls, $5 \mu \mathrm{l}$ of $10^{4}$ spores $/ \mathrm{ml}$ of 6 -day-old blastospores were drop inoculated on YMS media supplemented with hygromycin at $100 \mu \mathrm{g} / \mathrm{ml}$ (Neofroxx, Germany). YMS media without hygromycin was used as a control. Images were taken at $8 \mathrm{dpi}$. The experiment was repeated three times. 


\section{Comparative transcriptomic analysis}

RNA sequencing (RNA-seq) analysis was performed to identify differentially expressed genes in wild-type and melanin-deficient $\Delta z m r 1$ mutants. Roughly 100 blastospores of $Z$. tritici strains 3D1, 3D1 $\Delta z m r 1 \# 6,3 D 7$, and $3 \mathrm{D} 7 \Delta \mathrm{z} m r 1$ \#48 were placed onto PDA plates and incubated at $22{ }^{\circ} \mathrm{C}$ in the dark with $70 \%$ humidity. After 7 days, individual colonies were picked carefully from the plates using sterile forceps, collected, and frozen in liquid nitrogen. Three independent biological replicates were collected. The colonies were then homogenized using a Bead Ruptor with a cooling unit (Omni International) and zirconium oxide beads $(1.4 \mathrm{~mm})$. RNA was extracted using the GENEzol reagent (Geneaid Biotech) following the manufacturer's recommendations. On column DNAase, treatment was performed using RNeasy mini kit (Qiagen) following the manufacturer's instructions.

RNA-seq was performed on an Illumina HiSeq 2500 using paired-end reads at $2 \times 101 \mathrm{bp}$ as previously described [1]. Raw RNA-seq reads were trimmed using Trimmomatic v. 0.33 [90]. Trimmed reads were aligned to the Z. tritici parental genome 3D7 or 3D1 and transcriptome using TopHat v 2.0.13 [91]. Gene counts were calculated using HTSeq v0.6.1 [92] and differential gene expression analysis was performed using the $\mathrm{R}$ package EdgeR version 3.2.3 [93]. Relative RNA levels in the RNA-seq experiment were calculated by TMM (trimmed mean of $M$ values) normalization [94]. Mean TMM-normalized $\log _{2}$ CPM (counts per million mapped reads) were calculated for all the annotated genes. To identify differentially expressed genes between a wild-type strain and melanin-deficient $\Delta z m r 1$ knockouts, Benjamin-Hochberg false discovery rates (FDR) and an FDR-adjusted $p$ value were calculated. The RNAseq was deposited in SRA database with the accession number SRP143580 (https://www.ncbi.nlm.nih. gov/sra/SRP143580).

\section{Quantitative reverse transcription PCRs (qRT-PCRs)}

Expression levels of $Z m r 1$ in different $Z$. tritici strains and genetically modified strains were quantified using qRT-PCR. RNA was extracted from Z. tritici strains grown in vitro and harvested at 7 or 9 dpi depending on the experiment, as described earlier for the RNA-seq analysis. cDNA was synthesized from $500 \mathrm{ng}$ of RNA using oligo $(\mathrm{dT})_{18}$ primers and Revert Aid RT Reverse Transcription kit (Thermo Scientific) following the supplier's instructions. qRT-PCR analysis was performed using a $10-\mu \mathrm{l}$ reaction mix with $1 \mu \mathrm{l}$ of cDNA. A negative control with RNA alone and water was also included. Specific primers spanning introns were designed for the targets $Z m r 1$ and $18 \mathrm{~S}$ ribosomal RNA to avoid the risk of genomic DNA contamination (Additional file 20). Crossing point (Cp) values were calculated using absolute quantification and the second derivative method provided by LightCycler 480 software version 1.5 (Roche Diagnostics Corp., Indianapolis, IN, USA). "Advanced Relative Quantification" method was used to analyze the fold change in expression of Zmr1 when compared to the wild-type strains. Advanced Relative Quantification method was also used for estimating the fold change in expression of Zmrl in different strains of $Z$. tritici compared to 3D1.

\section{In planta virulence assay}

The Z. tritici wild-type 3D7 strain and three independent 3D7 $\Delta z m r 1$ mutants lacking melanin were compared for their ability to infect the winter wheat (Triticum aestivum) variety Drifter (DSP Ltd., Delley, Switzerland). Two wheat seeds were sown in peat soil (Jiffy GO PP7, Tref, Moerdijk, The Netherlands) in $7 \times$ $7 \times 9$-cm plastic pots and grown in a greenhouse at $18{ }^{\circ} \mathrm{C}$ day and $15{ }^{\circ} \mathrm{C}$ night, with a 16 -h light cycle and $70 \%$ relative humidity. Plants were fertilized 10 days after sowing with $10 \mathrm{ml} 0.1 \%$ Wuxal Universaldünger (Maag AG, Switzerland) per pot. Twelve 17-day-old seedlings were spray-inoculated with $15 \mathrm{ml}$ of a blastospore suspension $\left(10^{6}\right.$ spores $\left./ \mathrm{ml}\right)$ containing $0.1 \%(v / v)$ of Tween 20 (Sigma Aldrich). Pots were placed under $100 \%$ humidity for 3 days by covering them with a plastic bag. The second leaf of each plant was collected at $21 \mathrm{dpi}$ and pycnidia density (pycnidia $/ \mathrm{cm}^{2}$ leaf) and percentage of leaf area covered by lesions (PLACL) was analyzed using automated image analysis which was manually verified [37].

\section{Annotation of transposable elements in Z. tritici strains and sequence alignment}

For Z. tritici strains IPO323, 3D1, 3D7, 1E4, and 1A5, full genome annotations were already available [39, 44, 95]. Synteny of the QTL between IPO323, 3D1 and 3D7 was analyzed using blastn and visualized using the $R$ package genoPlotR v.0.8.4 [96]. We annotated and masked repetitive elements for the remaining $128 \mathrm{Z}$. tritici strains using RepeatModeler version 1.0.8 as described earlier [39, 82]. Whole-genome Illumina sequencing data from the 128 strains was obtained from the NCBI Short Read Archive under BioProject ID numbers PRJNA178194 and PRJNA327615 [55, 97-99]. We masked the genomes using RepeatMasker version 4.0.5 with the library previously obtained for Z. tritici strain IPO323 [38] according to the transposable element nomenclature defined by Wicker et al. [100]. Multiple sequence alignment of $Z m r 1$ in the Z. tritici strains was performed using AliView version 1.22 [101]. Amino acid sequence identity of $Z \mathrm{mr} 1$ in the $Z$. tritici strains was calculated using the Sequence Identities and Similarities (SIAS) [102]. 


\section{Statistical analysis}

Data analyses and plotting were performed using $\mathrm{R}$ version 3.3.1 and RStudio version 1.0.143 [103, 104] and Microsoft Excel. The non-parametric Kruskal-Wallis test was used to compare gray values between different strains/groups, since the data sets do not follow a normal distribution. Tukey's HSD test was used to estimate significant differences in pycnidial density between different $Z$. tritici strains for the in planta virulence assay. ANCOVA analysis was performed to determine significant differences in growth rates of $\Delta z m r 1$ mutants and the respective wild-type. Number of replicates and sizes of the samples are indicated in Additional file 21 and in the tables that show the results of the experiments. Data were represented using bar plots showing mean values and standard errors of the mean and boxplots.

\section{Additional files}

Additional file 1: $3 D 7$ accumulates more melanin than 3D1. Means and standard errors of gray values ( $0=$ black, $255=$ white) of at least 60 colonies of Z. tritici strains $3 \mathrm{D} 1$ and $3 \mathrm{D} 7$ at 7-12 days post inoculation (dpi). The experiment was performed three times with similar results. Asterisks indicate significant differences between 3D1 and 3D7 at each time point according to Kruskal-Wallis test ( $p$ values $\leq 0.05$ ). $n=$ number of colonies analyzed. (PDF $110 \mathrm{~kb}$ )

Additional file 2: Comparison of the QTLs obtained using the genome of the reference strain IPO323 and of the parental strain 3D7. (PDF $32 \mathrm{~kb}$ )

Additional file 3: Genes in the 95\% confidence interval of the QTL obtained using the genome of the parental strain 3D7. Genes in the Pks 1 gene cluster are indicated in bold. The total number of synonymous (Syn) and non-synonymous (Non-Syn) SNPs between both parental strains is indicated. Insertions upstream of the coding sequence are also indicated. (PDF $35 \mathrm{~kb}$ )

Additional file 4: $Z m r 1$ expression levels are lower in 3D1 than in 3D7 at 7 days post inoculation. Mean and standard errors (se) of the relative quantification $(\mathrm{RQ}$, fold change in expression level of $Z m r 1$ with respect to the $Z m r 1$ expression levels of the strain 3D1 at 7 days post inoculation, dpi) of the expression of $\mathrm{Zmr} 1$ in 3D1 and 3D7 at 7 and 9 dpi. The experiment was performed three times with similar results. (PDF $31 \mathrm{~kb}$ )

Additional file 5: Zmr1 regulates melanin biosynthesis in Z. tritici. (A) Three independent Zmr1 disruptant mutants in the 3D1 (\#46, \#48, and $\# 2.1)$ and in the 3D7 (\#3, \#6, and \#100) backgrounds lack melanin. Pictures of 10-day-old wild-type 3D7 and 3D7 $\Delta z m r 1$ colonies. (B) Melanized and albino pycnidia of 3D7 and 3D7 $\Delta z m r 1$, respectively, on wheat leaves of the cultivar Drifter, $21 \mathrm{dpi}$. (C) Expression values of the genes in the DHN melanin biosynthesis pathway $(\mathrm{Pks} 1=$ polyketide synthase $1 ; \operatorname{Thr} 1=1,3,8$-trihydroxynaphthalene reductase; $Z \mathrm{mr} 1=$ Zymoseptoria melanin regulation 1; Ayg1 $=$ Homolog of Aspergillus yellowish green) for the wild-type and $\Delta z m r 1$ in the 3D1 and 3D7 backgrounds, respectively. Mean of TMM (trimmed mean of $M$ values) normalized $\log _{2}$ CPM (counts per million mapped reads) values of three independent replicates with their standard deviation are plotted. Asterisks indicate statistical differences between the wild-type and the mutant ( $p$ value $\leq 0.05, F D R \leq 0.05$ ). (TIF $1614 \mathrm{~kb}$ )

Additional file 6: Reduced expression of genes in the Pks 1 cluster in $\Delta z m r 1$ mutants. Mean and standard error (se) of CPM (counts per million mapped reads) values of genes significantly downregulated (false discovery rates, $F D R \leq 0.05)$ in both $\Delta z m r 1$ mutants compared to the wild-type strains 3D1 and 3D7. Means and standard errors of the mean of three independent replicates are indicated. Genes previously shown to be involved in melanin biosynthesis are shown in bold. (PDF $423 \mathrm{~kb}$ )
Additional file 7: Sequence variation in $Z m r 1$ promoter contributes to differences in melanin accumulation. Means and standard errors of gray values ( $0=$ black, $255=$ white) of 3D1, 3D7, 3D7 $\Delta z m r 1,3 D 7 \Delta z m r 1+$ $\mathrm{Zmrl}_{3 \mathrm{D} 1}$, and $3 \mathrm{D} 7 \Delta \mathrm{Zmr1}+\mathrm{Zmr}_{3 \mathrm{D} 7}, 7$ days post inoculation (dpi) based on at least 20 colonies. Asterisks $(*)$ and pluses $(+)$ indicate significant differences in mean gray values of each strain with respect to the mean gray value of $3 \mathrm{D} 7$ and $3 \mathrm{D} 7 \Delta z \mathrm{mr}$, respectively (Kruskal-Wallis, $p$ values $\leq 0.05$ ). The experiment was performed twice with similar results. $\mathrm{NA}=$ not applicable. (PDF $335 \mathrm{~kb}$ )

Additional file 8: The transposable element insertion upstream of $\mathrm{Zmr} 1$ in 3D1 downregulates $Z m r 1$ expression. Mean gray values $(0=$ black, 255 $=$ white) based on at least 35 colonies of the wild-types 3D1 and 3D7, three independent TE deletion mutants in the 3D1 background (3D1 $\triangle T E$ \#93, \#131, \#239) and the two ectopic controls (3D1+ Hyg \#3 and \#6), 7 days post inoculation. Asterisks $\left(^{*}\right)$ indicate that the strains are significantly darker than the wild-type 3D1 (Kruskal-Wallis, $p$ value $\leq 0.05$ ). The experiment was performed three times with 3D1 $\Delta$ TE \#93 and twice with \#131 and \#239. (PDF $330 \mathrm{~kb}$ )

Additional file 9: Non-melanized mutants grow faster than the wildtypes. Radial growth rates (slope of the curves) of wild-type strains and melanin-deficient mutants (3D1 $\Delta z m r 1$ and $3 D 7 \Delta z m r 1)$ were obtained by plotting radial size $(\mathrm{mm})$ of the colonies over time. The fit of the radial growth curve estimated using a linear model was evaluated using Pearson's correlation coefficient ( $r^{2}$ value). Asterisks $(*)$ indicate significant differences in growth rate (slope) between the wild-type and the mutant according to ANCOVA analysis ( $p$ values $\leq 0.05$ ). The experiment was performed three times with similar results. (PDF $332 \mathrm{~kb}$ )

Additional file 10: Colonies of non-melanized mutants are bigger than those of the corresponding wild-types. Means and standard errors of the radial size $(\mathrm{mm})$ based on at least 20 colonies at different days post inoculation (dpi). The experiment was performed three times with similar results. (PDF $65 \mathrm{~kb}$ )

Additional file 11: Melanin is not essential for virulence. All three $3 \mathrm{D} 7 \Delta z m r 1$ mutants were equally virulent on the winter wheat variety Drifter, compared to the wild-type 3D7 as indicated by pycnidia/ $\mathrm{cm}^{2}$ leaf 21 days post infection (Turkey's HSD test, $p$ value $\leq 0.05$ ). Mean and standard error of the mean of 12 independent leaves are shown. The experiment was performed three times for line \#6 with similar results. (TIF $86 \mathrm{~kb}$ )

Additional file 12: Melanin-deficient mutants are not impaired in virulence. Mean of percentage of leaf area covered by lesions (PLACL)

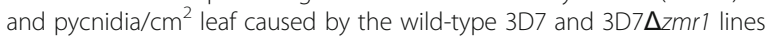
on the wheat cultivar Drifter, at 22 days post inoculation. Mean and standard error of the mean of 12 leaves are shown. The experiment was performed twice with the line 6 and 3D7 obtaining similar results. No statistical differences in PLACL and pycnidia/ $\mathrm{cm}^{2}$ leaf were detected between 3D7 and 3D7 $\Delta \mathrm{mr} 1$ according to Tukey's HSD (honest significant difference) test ( $p$ values $\leq 0.05$ ). (PDF $223 \mathrm{~kb}$ )

Additional file 13: Melanin protects $Z$. tritici against SDHI fungicides. Reduction in growth by fungicides (\% growth decrease) of the wild-type $3 D 7$ and $3 D 7 \Delta z m r 1$. Strains were grown on Whatman filter paper placed on PDA for 5 days and later transferred to PDA plates supplemented with the fungicide bixafen or propiconazole. Asterisks $\left(^{*}\right)$ indicate that the percentage in decrease in growth of the mutant strain, in the presence of the fungicide, is significantly different from the wild-type 3D7 (KruskalWallis, $p$ value $\leq 0.05$ ). Mean and standard error of differential radial size of at least 25 colonies grown on three independent plates are presented. The experiment was performed twice with similar results. (PDF $122 \mathrm{~kb}$ )

Additional file 14: No insertions of transposable elements were identified upstream of $\mathrm{Zmr} 1$ in IPO323. A) Melanization levels of the reference strain IPO323 from 8 to 12 days post inoculation. Bars represent means and standard errors of the mean gray value based on at least 60 colonies. Representative pictures of both strains are shown below the bar plot for all the time points. The experiment was performed two times with similar results. Gray value scale $(0=$ black, $255=$ white $)$ is shown on the left. B) Synteny plot of the QTL between 3D7, 3D1 and the reference strain IPO323 showing the insertion of an island of transposable elements $1.8 \mathrm{~kb}$ upstream of the start codon of $\mathrm{Zmrl}$ only in 3D1. Brown lines indicate collinear sequences. The positions of genes and transposable 
elements are shown using purple arrows and blue bars, respectively. Vertical yellow lines indicate simple repeats. The different shades of blue represent different classes of transposable elements that were classified according to the three-letter code described in Wicker et al., [100]. The first letter indicates the class ( $R=$ RNA class and $D=D N A$ class), the second letter indicates the order $(L=L T R, I=L i n e, T=T I R)$ and the third letter indicates the superfamily ( $\mathrm{G}=$ Gypsy, I = I, X = unknown). (TIF $100521 \mathrm{~kb}$ )

Additional file 15: SNPs in Zmr1 regulatory sequences. Alignment of the nucleotide sequences 1862 bp upstream of the coding sequence of Zmr1 in 3D1 and 3D7. Vertical pink bars indicate the 12 SNPs in the promoter region. Frequency (in percentage) of the 3D1 and 3D7 variants in the analyzed 132 strains of $Z$. tritici are indicated. (TIF $16001 \mathrm{~kb}$ )

Additional file 16: No significant difference in the distribution of mean gray values of different $Z$. tritici strains belonging to four different populations. Distribution of mean gray values of different $Z$. tritici strains from four different populations across the world (3 strains from Australia, 8 from Switzerland, 9 from Israel, and 13 from the USA). The colors of the violin plot indicate different populations. Black dots represent individual data points, which correspond to the mean gray values of each $Z$. tritici strain at 7 days post inoculation. At least 100 colonies grown on five different plates were evaluated. The experiment was performed three times with similar results. No statistically significant differences were observed between the populations (Kruskal-Wallis test, $p$ value $\leq 0.05$ ). (TIF $172 \mathrm{~kb}$ )

Additional file 17: Melanization and Zmr1 expression levels in Z. tritici strains from around the world. Means and standard errors of the mean of gray values ( $0=$ black, $255=$ white) and of $Z m r 1$ expression at 7 days post inoculation. $n$ indicates the number of colonies analyzed. Gray values were measured in three independent experiments, and similar results were obtained. Expression analysis was performed twice and provided similar results. AUS: Australia, CH: Switzerland, ISY: Israel and OR: USA (Oregon), $\mathrm{TE}=$ transposable element, $\mathrm{P}=$ Present, $\mathrm{A}=$ Absent. $(\mathrm{PDF} 205 \mathrm{~kb})$

Additional file 18: Altered growth morphology of $3 D 7 \Delta z m r 1$ mutants on YMS but not in PDA. Morphology of 3D1, 3D7, and the mutants in Zmrl grown on YMS and PDA at 7 days post inoculation. (TIF $6379 \mathrm{~kb}$ )

Additional file 19: Generation of mutants (A) Schematic diagram showing the location of primers used for generating Zmr1 disruptant mutants $(\mathrm{U} 2+\mathrm{U} 3, \mathrm{D} 1+\mathrm{D} 2)$ and the primers used for screening the transformants (U1 + Hyg UF, Hyg DF + D3). (B) Schematic diagram showing the location of primers used for generating the transposable element (TE) deletion mutants in 3D1 background ( $\triangle T$ TE; TE_U2 + TE_U3, TE_D1 + TE_D2) and the primers used for screening the transformants (TE_U1 + Hyg UF, Hyg DF + TE_D3). (C) Diagrams showing the location of primers used for generating the transformants expressing $Z m r l$ gene in

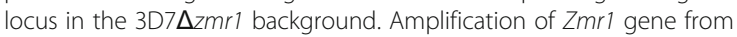
genomic DNA of 3D1 and 3D7 strains was performed using Zmr1 F and Zmrl R primers; the geneticin resistance cassette (Gen) was amplified from pCGEN with Gen F and Gen R. Both amplicons were fused to the pES1 backbone to generate an intermediate construct, which was used to amplify $Z m r l$ and the geneticin resistance cassette using $Z m r 1$ in locus $\mathrm{F}+\mathrm{Zmr} 1$ in locus $\mathrm{R}$. Up-flanking and down-flanking regions of the insertion site were amplified from 3D7 genomic DNA using the primers UF_F + UF_R and DF_F + DF_R. Primers used for screening the transformants (P1 + P2, P3 + P4) are also shown. $L B$ and RB indicates the left and right border of the binary vector $\mathrm{pES1}$. Hyg.R = hygromycin resistance cassette. (PDF $139 \mathrm{~kb}$ )

Additional file 20: List of primers used in the study, their sequence, and their purpose. (PDF $287 \mathrm{~kb}$ )

Additional file 21: Sample size and number of independent replicates performed in each experiment. In the experiments where gray value and radial growth were measured, $n$ indicates the lowest number of colonies per strain analyzed in the experiment. In the experiments where expression levels of $\mathrm{Zmrl}$ were measured, $n$ indicates the number of technical replicates. (PDF $113 \mathrm{~kb}$ )

\section{Abbreviations}

Pks1: Polyketide synthase 1; QTL: Quantitative trait locus; SNP: Single nucleotide polymorphism; Zmr1: Zymoseptoria melanin regulation 1

\section{Acknowledgements}

RNA sequencing was performed at the quantitative genomics facility of the D-BSSE, ETH Zurich. GPCR was performed at the Genetic Diversity Centre (GDC), ETH Zurich. Technical assistance was provided by Lotta Köppel. Eva H. Stukenbrock and Jason J. Rudd provided us with vectors and Gert H.J. Kema with IPO323 strain.

\section{Funding}

The research was supported by the Swiss National Science Foundation (grant 31003A_155955, http://www.snf.ch/en/Pages/default.aspx.) and by the ETH Zurich Research Commission (grant 12-03, https://www.ethz.ch/en/research/research-promotion/eth-internal-programmes/eth-grants.html) to BAM. PK was supported by the Swiss State Secretariat under the Education Research and Innovation, through the Federal Commission for Scholarships for Foreign Students. CP was supported by an INRA Young Scientist grant. DC is supported by the Swiss National Science Foundation (grant 31003A_173265, http://www.snf.ch/en/Pages/default.aspx). The funders had no role in the design of the study, data collection, analysis and interpretation of data, and in writing the manuscript.

\section{Availability of data and materials}

The datasets generated and analyzed during the current study are available in the SRA database (accession number: SRP143580), https:// www.ncbi.n/m.nih.gov/sra/SRP143580.

\section{Authors' contributions}

PK contributed to the conception and design of the analysis, acquisition of data, analysis of the data, and writing of the original draft of the manuscript. LM contributed to the design of the analysis and acquisition of data and contributed to the edition and revision of the manuscript. XM analyzed the RNAseq data and contributed to the edition of the manuscript. CP contributed to the analysis of the transposable elements in the populations. FEH contributed to the bioinformatics analysis of transposable element variation in the populations and edited the manuscript. DC contributed to the analysis of the transposable elements in the populations and edited and reviewed the manuscript. BAM acquired funding and reviewed the manuscript. ASV contributed to the conception and design of the analysis, supervision and analysis of the data, and writing of the manuscript. All authors read and approved the final manuscript.

\section{Ethics approval and consent to participate}

Not applicable

\section{Consent for publication}

Not applicable

\section{Competing interests}

The authors declare that they have no competing interests.

\section{Publisher's Note}

Springer Nature remains neutral with regard to jurisdictional claims in published maps and institutional affiliations.

\section{Author details}

${ }^{1}$ Plant Pathology, Institute of Integrative Biology, ETH Zurich, Zurich, Switzerland. ${ }^{2}$ UMR BIOGER, INRA, AgroParisTech, Université Paris-Saclay, Thiverval-Grignon, France. ${ }^{3}$ Ecologie Systématique Evolution, Univ. Paris-Sud, AgroParisTech, CNRS, Université Paris Saclay, Orsay, France. ${ }^{4}$ Laboratory of Evolutionary Genetics, Institute of Biology, University of Neuchâtel, Neuchâtel, Switzerland.

Received: 6 May 2018 Accepted: 20 June 2018

Published online: 16 July 2018

\section{References}

1. Palma-Guerrero J, Ma X, Torriani SFF, Zala M, Francisco CS, Hartmann FE, et al. Comparative transcriptome analyses in Zymoseptoria tritici reveal significant differences in gene expression among strains during plant infection. Mol Plant-Microbe Interact. 2017;30:231-44.

2. Romero DA, Hasan AH, Lin Y, Kime L, Ruiz-Larrabeiti O, Urem M, et al. A comparison of key aspects of gene regulation in Streptomyces coelicolor and 
Escherichia coli using nucleotide-resolution transcription maps produced in parallel by global and differential RNA sequencing: RNA-seq comparison of S coelicolor and E coli. Mol Microbiol. 2014;94:963-87.

3. Whitehead A, Crawford DL. Variation in tissue-specific gene expression among natural populations. Genome Biol. 2005;6:R13.

4. Bhullar NK, Zhang Z, Wicker T, Keller B. Wheat gene bank accessions as a source of new alleles of the powdery mildew resistance gene Pm3: a large scale allele mining project. BMC Plant Biol. 2010;10:88.

5. Spiess B, Postina P, Reinwald M, Cornely OA, Hamprecht A, Hoenigl M, et al. Incidence of Cyp51 A key mutations in Aspergillus fumigatus -a study on primary clinical samples of immunocompromised patients in the period of 1995-2013. PLoS One. 2014;9:e103113.

6. Wray GA. The evolutionary significance of cis-regulatory mutations. Nat Rev Genet. 2007:8:206-16.

7. Ali S, Laurie JD, Linning R, Cervantes-Chávez JA, Gaudet D, Bakkeren G. An immunity-triggering effector from the Barley smut fungus Ustilago hordei resides in an Ustilaginaceae-specific cluster bearing signs of transposable element-assisted evolution. PLoS Pathog. 2014;10:e1004223.

8. Druka A, Potokina E, Luo Z, Bonar N, Druka I, Zhang L, et al. Exploiting regulatory variation to identify genes underlying quantitative resistance to the wheat stem rust pathogen Puccinia graminis f. sp. tritici in barley. Theor Appl Genet. 2008;117:261-72.

9. Belting HG, Shashikant CS, Ruddle FH. Modification of expression and cis-regulation of Hoxc8 in the evolution of diverged axial morphology. Proc Natl Acad Sci U S A. 1998;95:2355-60

10. Ludwig MZ, Kreitman M. Evolutionary dynamics of the enhancer region of even-skipped in Drosophila. Mol Biol Evol. 1995;12:1002-11.

11. Rockman MV, Wray GA. Abundant raw material for cis-regulatory evolution in humans. Mol Biol Evol. 2002;19:1991-2004.

12. Streelman JT, Kocher TD. Microsatellite variation associated with prolactin expression and growth of salt-challenged tilapia. Physiol Genomics. 2002;9:1-4.

13. Trefilov A, Berard J, Krawczak M, Schmidtke J. Natal dispersal in rhesus macaques is related to serotonin transporter gene promoter variation. Behav Genet. 2000;30:295-301.

14. Tournamille C, Colin Y, Cartron JP, Le Van Kim C. Disruption of a GATA motif in the Duffy gene promoter abolishes erythroid gene expression in Duffynegative individuals. Nat Genet. 1995;10:224-8.

15. Wang C, Yang Q, Wang W, Li Y, Guo Y, Zhang D, et al. A transposondirected epigenetic change in ZmCCT underlies quantitative resistance to Gibberella stalk rot in maize. New Phytol. 2017;215:1503-15.

16. Girard L, Freeling M. Regulatory changes as a consequence of transposon insertion. Dev Genet. 1999;25:291-6.

17. Kang S, Lebrun MH, Farrall L, Valent B. Gain of virulence caused by insertion of a Pot3 transposon in a Magnaporthe grisea avirulence gene. Mol PlantMicrobe Interact. 2001;14:671-4.

18. Omrane S, Audéon C, Ignace A, Duplaix C, Aouini L, Kema G, et al. Plasticity of the MFS1 promoter leads to multidrug resistance in the wheat pathogen Zymoseptoria tritici. mSphere. 2017;2:e00393-17.

19. Mao H, Wang H, Liu S, Li Z, Yang X, Yan J, et al. A transposable element in a NAC gene is associated with drought tolerance in maize seedlings. Nat Commun. 2015;6:8326.

20. Yu A, Lepère G, Jay F, Wang J, Bapaume L, Wang Y, et al. Dynamics and biological relevance of DNA demethylation in Arabidopsis antibacterial defense. Proc Natl Acad Sci U S A. 2013;110:2389-94.

21. Seidl MF, Cook DE, Thomma BPHJ. Chromatin biology impacts adaptive evolution of filamentous plant pathogens. PLoS Pathog. 2016;12: e1005920-8.

22. Seidl MF, Thomma BPHJ. Transposable elements direct the coevolution between plants and microbes. Trends Genet. 2017;33:842-51.

23. Dong S, Raffaele S, Kamoun S. The two-speed genomes of filamentous pathogens: waltz with plants. Curr Opin Genet Dev. 2015;35:57-65.

24. Raffaele S, Kamoun S. Genome evolution in filamentous plant pathogens: why bigger can be better. Nat Publ Group. 2012;10:417-30.

25. Croll D, McDonald BA. The genetic basis of local adaptation for pathogenic fungi in agricultural ecosystems. Mol Ecol. 2016;26:2027-40.

26. Giraud T, Gladieux P, Gavrilets S. Linking the emergence of fungal plant diseases with ecological speciation. Trends Ecol Evol. 2010;25:387-95.

27. Prentis PJ, Wilson JRU, Dormontt EE, Richardson DM, Lowe AJ. Adaptive evolution in invasive species. Trends Plant Sci. 2008;13:288-94.

28. Chumley FG. Genetic analysis of melanin-deficient, non-pathogenic mutants of Magnaporthe grisea. Mol Plant-Microbe Interact. 1990;3:135.
29. Jahn B, Koch A, Schmidt A, Wanner G, Gehringer H, Bhakdi S, et al. Isolation and characterization of a pigmentless-conidium mutant of Aspergillus fumigatus with altered conidial surface and reduced virulence. Infect Immun. 1997:65:5110-7.

30. Liu GY, Nizet V. Color me bad: microbial pigments as virulence factors. Trends Microbiol. 2009:17:406-13.

31. Singaravelan N, Grishkan I, Beharav A, Wakamatsu K, Ito S, Nevo E. Adaptive melanin response of the soil fungus Aspergillus niger to UV radiation stress at "Evolution Canyon", Mount Carmel, Israel. PLoS One. 2008;3:e2993-5.

32. Bell $\mathrm{AA}$, Wheeler MH. Biosynthesis and functions of fungal melanins. Annu Rev Phytopathol. 1986;24:411-51.

33. Lendenmann MH, Croll D, Stewart EL, McDonald BA. Quantitative trait locus mapping of melanization in the plant pathogenic fungus Zymoseptoria tritici. G3 Bethesda Md. 2014;4:2519-33.

34. Pal AK, Gajjar DU, Vasavada AR. DOPA and DHN pathway orchestrate melanin synthesis in Aspergillus species. Med Mycol. 2014;52:10-8.

35. Lendenmann MH, Croll D, Palma-Guerrero J, Stewart EL, McDonald BA. QTL mapping of temperature sensitivity reveals candidate genes for thermal adaptation and growth morphology in the plant pathogenic fungus Zymoseptoria tritici. Heredity. 2016;116:384-94.

36. Hartmann FE, Sanchez-Vallet A, McDonald BA, Croll D. A fungal wheat pathogen evolved host specialization by extensive chromosomal rearrangements. ISME J. 2017;11:1189-204.

37. Stewart EL, Croll D, Lendenmann MH, Sanchez-Vallet A, Hartmann FE, Palma-Guerrero J, et al. Quantitative trait locus mapping reveals complex genetic architecture of quantitative virulence in the wheat pathogen Zymoseptoria tritici. Mol Plant Pathol. 2017;19:201-16.

38. Grandaubert J, Bhattacharyya A, Stukenbrock EH. RNA-seq-based gene annotation and comparative genomics of four fungal grass pathogens in the genus Zymoseptoria identify novel orphan genes and species-specific invasions of transposable elements. G3 Bethesda Md. 2015;5:1323-33.

39. Plissonneau C, Stürchler A, Croll D. The evolution of orphan regions in genomes of a fungal pathogen of wheat. MBio. 2016;7(5):e01231-16.

40. Schotanus K, Soyer JL, Connolly LR, Grandaubert J, Happel P, Smith KM, et al. Histone modifications rather than the novel regional centromeres of Zymoseptoria tritici distinguish core and accessory chromosomes. Epigenetics Chromatin. 2015;8:41

41. Lendenmann MH, Croll D, McDonald BA. QTL mapping of fungicide sensitivity reveals novel genes and pleiotropy with melanization in the pathogen Zymoseptoria tritici. Fungal Genet Biol. 2015;80:53-67.

42. Tsuji G, Kenmochi Y, Takano Y, Sweigard J, Farrall L, Furusawa I, et al. Novel fungal transcriptional activators, $\mathrm{Cmr} 1 \mathrm{p}$ of Colletotrichum lagenarium and pig1p of Magnaporthe grisea, contain Cys2His2 zinc finger and Zn(II)2Cys6 binuclear cluster DNA-binding motifs and regulate transcription of melanin biosynthesis genes in a developmentally specific manner. Mol Microbiol. 2000;38:940-54.

43. Nosanchuk JD, Casadevall A. The contribution of melanin to microbial pathogenesis. Cell Microbiol. 2003;5:203-23.

44. Goodwin SB, Ben M'Barek S, Dhillon B, AHJ W, Crane CF, Hane JK, et al. Finished genome of the fungal wheat pathogen Mycosphaerella graminicola reveals dispensome structure, chromosome plasticity, and stealth pathogenesis. PLoS Genet. 2011;7:e1002070.

45. Doebley JF, Gaut BS, Smith BD. The molecular genetics of crop domestication. Cell. 2006;127:1309-21.

46. Indjeian VB, Kingman GA, Jones FC, Guenther CA, Grimwood J, Schmutz J, et al. Evolving new skeletal traits by cis-regulatory changes in bone morphogenetic proteins. Cell. 2016;164:45-56.

47. Gompel N, Prud'homme B, Wittkopp PJ, Kassner VA, Carroll SB. Chance caught on the wing: cis-regulatory evolution and the origin of pigment patterns in Drosophila. Nature. 2005:433:481-7.

48. Prud'homme B, Gompel N, Carroll SB. Emerging principles of regulatory evolution. Proc Natl Acad Sci. 2007;104:8605-12.

49. Brem RB. Genetic dissection of transcriptional regulation in budding yeast. Science. 2002;296:752-5.

50. Chang J, Zhou Y, Hu X, Lam L, Henry C, Green EM, et al. The molecular mechanism of a cis-regulatory adaptation in yeast. PLoS Genet. 2013;9: e1003813.

51. Tsong AE, Miller MG, Raisner RM, Johnson AD. Evolution of a combinatorial transcriptional circuit. Cell. 2003;115:389-99.

52. Inmels J. Rewiring of the yeast transcriptional network through the evolution of motif usage. Science. 2005;309:938-40. 
53. Salinas F, de Boer CG, Abarca V, García V, Cuevas M, Araos S, et al. Natural variation in non-coding regions underlying phenotypic diversity in budding yeast. Sci Rep. 2016;6:21849.

54. Smith EN, Kruglyak L. Gene-environment interaction in yeast gene expression. PLoS Biol. 2008;6:e83.

55. Mohd-Assaad N, McDonald BA, Croll D. Multilocus resistance evolution to azole fungicides in fungal plant pathogen populations. Mol Ecol. 2016;25: 6124-42.

56. Antoniazza S, Burri R, Fumagalli L, Goudet J, Roulin A. Local adaptation maintains clinal variation in melanin-based coloration of European barn owls (Tyto alba). Evolution. 2010;64:1944-54.

57. Tang H, Barsh GS. Skin color variation in Africa. Science. 2017;358:867-8.

58. Kawamura C, Moriwaki J, Kimura N, Fujita Y, Fuji S, Hirano T, et al. The melanin biosynthesis genes of Alternaria alternata can restore pathogenicity of the melanin-deficient mutants of Magnaporthe grisea. Mol Plant-Microbe Interact. 1997;10:446-53.

59. Zhdanova NM, Zakharchenko VO, Vasilevs'ka AY, Skol'nij OT, Nakonechna LT, Artishkova LV. Peculiarities of soil mycobiota composition in Chernobyl NPP. Ukrayinskij Bot Zhurnal. 1994;51:134-44.

60. Wang Y, Casadevall A. Susceptibility of melanized and non-melanized Cryptococcus neoformans to nitrogen and oxygen derived oxidants. Infect Immun. 1994;62:3004-7.

61. van Duin D, Casadevall A, Nosanchuk JD. Melanization of Cryptococcus neoformans and Histoplasma capsulatum reduces their susceptibilities to amphotericin B and caspofungin. Antimicrob Agents Chemother. 2002;46: 3394-400.

62. Nosanchuk JD, Casadevall A. Impact of melanin on microbial virulence and clinical resistance to antimicrobial compounds. Antimicrob Agents Chemother. 2006;50:3519-28

63. Chujo T, Scott B. Histone H3K9 and H3K27 methylation regulates fungal alkaloid biosynthesis in a fungal endophyte-plant symbiosis. Mol Microbiol. 2014;92:413-34.

64. Connolly LR, Smith KM, Freitag M. The Fusarium graminearum histone $\mathrm{H3}$ K27 methyltransferase KMT6 regulates development and expression of secondary metabolite gene clusters. PLoS Genet. 2013;9:e1003916.

65. Eichten SR, Ellis NA, Makarevitch I, Yeh C-T, Gent JI, Guo L, et al. Spreading of heterochromatin is limited to specific families of maize retrotransposons. PLoS Genet. 2012:8:e1003127.

66. Galazka JM, Freitag M. Variability of chromosome structure in pathogenic fungi of "ends and odds". Curr Opin Microbiol. 2014;20:19-26.

67. Lewis ZA, Honda S, Khlafallah TK, Jeffress JK, Freitag M, Mohn F, et al. Relics of repeat-induced point mutation direct heterochromatin formation in Neurospora crassa. Genome Res. 2009;19:427-37.

68. Hollister JD, Gaut BS. Epigenetic silencing of transposable elements: a tradeoff between reduced transposition and deleterious effects on neighboring gene expression. Genome Res. 2009;19:1419-28.

69. Le TN, Miyazaki Y, Takuno S, Saze H. Epigenetic regulation of intragenic transposable elements impacts gene transcription in Arabidopsis thaliana. Nucleic Acids Res. 2015:43:3911-21.

70. Lee YCG, Karpen GH. Pervasive epigenetic effects of Drosophila euchromatic transposable elements impact their evolution. elife. 2017:6:e25762

71. Song X, Cao X. Transposon-mediated epigenetic regulation contributes to phenotypic diversity and environmental adaptation in rice. Curr Opin Plant Biol. 2017;36:111-8.

72. Bucher E, Reinders J, Mirouze M. Epigenetic control of transposon transcription and mobility in Arabidopsis. Curr Opin Plant Biol. 2012;15:503-10.

73. Makarevitch I, Waters AJ, West PT, Stitzer M, Hirsch CN, Ross-Ibarra J, et al. Transposable elements contribute to activation of maize genes in response to abiotic stress. PLoS Genet. 2015;11:e1004915.

74. Wessler SR. Plant retrotransposons: turned on by stress. Curr Biol. 1996;6:959-61.

75. Soyer JL, Rouxel T, Fudal I. Chromatin-based control of effector gene expression in plant-associated fungi. Curr Opin Plant Biol. 2015;26:51-6.

76. Faino L, Seidl MF, Shi-Kunne X, Pauper M, van den Berg GCM, Wittenberg AHJ, et al. Transposons passively and actively contribute to evolution of the twospeed genome of a fungal pathogen. Genome Res. 2016;26(8):1091-100.

77. Soyer JL, El Ghalid M, Glaser N, Ollivier B, Linglin J, Grandaubert J, et al. Epigenetic control of effector gene expression in the plant pathogenic fungus Leptosphaeria maculans. PLoS Genet. 2014;10:e1004227-19.

78. Garin G, Fournier C, Andrieu B, Houlès V, Robert C, Pradal C. A modelling framework to simulate foliar fungal epidemics using functional-structural plant models. Ann Bot. 2014;114:795-812.
79. Ma LJ, van der Does HC, Borkovich KA, Coleman JJ, Daboussi MJ, Di Pietro A, et al. Comparative genomics reveals mobile pathogenicity chromosomes in Fusarium. Nature. 2010;464:367-73.

80. Zhan J, Pettway RE, McDonald BA. The global genetic structure of the wheat pathogen Mycosphaerella graminicola is characterized by high nuclear diversity, low mitochondrial diversity, regular recombination, and gene flow. Fungal Genet Biol. 2003;38:286-97.

81. Zhan J, Linde CC, Jurgens T, Merz U, Steinebrunner F, McDonald BA. Variation for neutral markers is correlated with variation for quantitative traits in the plant pathogenic fungus Mycosphaerella graminicola. Mol Ecol. 2005:14:2683-93.

82. Linde CC, Zhan J, McDonald BA. Population structure of Mycosphaerella graminicola: from lesions to continents. Phytopathology. 2002:92:946-55.

83. Meile L, Brunner PC, Plissonneau C, Hartmann FE, BA MD, Sánchez-Vallet A. A fungal avirulence factor encoded in a highly plastic genomic region triggers partial resistance to septoria tritici blotch. New Phytol. 2018. https:// doi.org/10.1111/nph.15180.

84. Arends D, Prins P, Jansen RC, Broman KW. R/qtl: high-throughput multiple QTL mapping. Bioinformatics. 2010;26:2990-2.

85. Schneider CA, Rasband WS, Eliceiri KW. NIH Image to ImageJ: 25 years of image analysis. Nat Methods. 2012;9:671-5.

86. Motteram J, Lovegrove A, Pirie E, Marsh J, Devonshire J, van de Meene A, et al. Aberrant protein $\mathrm{N}$-glycosylation impacts upon infection-related growth transitions of the haploid plant-pathogenic fungus Mycosphaerella graminicola. Mol Microbiol. 2011;81:415-33.

87. Lazo GR, Stein PA, Ludwig RA. A DNA transformation-competent Arabidopsis genomic library in Agrobacterium. Biotechnol Nat Publ Co. 1991;9:963-7.

88. Kilaru S, Schuster M, Latz M, Das Gupta S, Steinberg N, Fones H, et al. A gene locus for targeted ectopic gene integration in Zymoseptoria tritici. Fungal Genet Biol. 2015;79:118-24.

89. Zwiers LH, De Waard MA. Efficient Agrobacterium tumefaciens-mediated gene disruption in the phytopathogen Mycosphaerella graminicola. Curr Genet. 2001;39:388-93.

90. Bolger AM, Lohse M, Usadel B. Trimmomatic: a flexible trimmer for Illumina sequence data. Bioinformatics. 2014;30:2114-20.

91. Trapnell C, Pachter L, Salzberg SL. TopHat: discovering splice junctions with RNA-Seq. Bioinformatics. 2009:25:1105-11.

92. Anders S, Pyl PT, Huber W. HTSeq-a Python framework to work with highthroughput sequencing data. Bioinformatics. 2015;31:166-9.

93. Robinson MD, McCarthy DJ, Smyth GK. edgeR: a Bioconductor package for differential expression analysis of digital gene expression data. Bioinformatics. 2010;26:139-40.

94. Robinson MD, Oshlack A. A scaling normalization method for differential expression analysis of RNA-seq data. Genome Biol. 2010;11:R25.

95. Plissonneau C, Hartmann FE, Croll D. Pangenome analyses of the wheat pathogen Zymoseptoria tritici reveal the structural basis of a highly plastic eukaryotic genome. BMC Biol. 2018;16:5.

96. Guy L, Roat Kultima J, Andersson SGE. genoPlotR: comparative gene and genome visualization in R. Bioinformatics. 2010;26:2334-5.

97. Torriani SFF, Stukenbrock EH, Brunner PC, McDonald BA, Croll D. Evidence for extensive recent intron transposition in closely related fungi. Curr Biol. 2011;21:2017-22.

98. Croll D, Zala M, McDonald BA. Breakage-fusion-bridge cycles and large insertions contribute to the rapid evolution of accessory chromosomes in a fungal pathogen. PLoS Genet. 2013;9:e1003567.

99. Hartmann FE, Croll D. Distinct trajectories of massive recent gene gains and losses in populations of a microbial eukaryotic pathogen. Mol Biol Evol. 2017;34:2808-22.

100. Wicker T, Sabot F, Hua-Van A, Bennetzen JL, Capy P, Chalhoub B, et al. A unified classification system for eukaryotic transposable elements. Nat Rev Genet. 2007:8:973-82.

101. Larsson A. AliView: a fast and lightweight alignment viewer and editor for large datasets. Bioinformatics. 2014;30(22):3276-8.

102. Rech P. SIAS: sequence identities and similarities. 2008. Available from: http://imed.med.ucm.es/Tools/sias.html. Accessed 26 June 2018.

103. RStudio. Boston, MA; 2012. Available from: http://www.rstudio.org/. Accessed 26 June 2018.

104. R Core Team. R: a language and environment for statistical computing. Vienna, Austria: R Foundation for Statistical Computing; 2013. Available from: http://www.R-project.org/. Accessed 26 June 2018. 